Supporting Information

\title{
Electronic Structures and Properties of Twisted Polyacenes
}

Joseph E. Norton and K. N. Houk*

\section{Computational Details}

DFT. All calculations were carried out using the Gaussian 03 suite of programs. The hybrid density functional Becke3LYP ${ }^{1,2}$ with the 6-31G(d) basis set was used for all DFT calculations. Vertical $\mathrm{S}_{0}-\mathrm{S}_{1}$ transition energies were calculated using TDDFT (6-31G(d)) methods.

Geometry Optimizations. Minima were fully optimized under the specified twist angle restraint, defined as the dihedral angle formed by the two end carbons of each terminal benzene ring. Singlet-triplet gaps were determined by optimizing both the ground state singlet and excited state triplet electronic configurations. For singlet states, both spinrestricted and spin-unrestricted wavefunctions were calculated. Wavefunctions were tested for stability where necessary. Spin-contamination values and absolute electronic energies without application of a spin correction procedure are reported in the tables below.

(1) Becke, A. D. J. Chem. Phys. 1993, 98, 5648-5652.

(2) Lee, C.; Yang, W.; Parr, R. G. Phys. Rev. B: Condens. Matter Mater. Phys. 1988, 37, 785-789. 


\section{Software Citations}

Gaussian 03, Revision C.02, Frisch, M. J.; Trucks, G. W.; Schlegel, H. B.; Scuseria, G. E.; Robb, M. A.; Cheeseman, J. R.; Montgomery, J., J. A.; Vreven, T.; Kudin, K. N.; Burant, J. C.; Millam, J. M.; Iyengar, S. S.; Tomasi, J.; Barone, V.; Mennucci, B.; Cossi, M.; Scalmani, G.; Rega, N.; Petersson, G. A.; Nakatsuji, H.; Hada, M.; Ehara, M.; Toyota, K.; Fukuda, R.; Hasegawa, J.; Ishida, M.; Nakajima, T.; Honda, Y.; Kitao, O.; Nakai, H.; Klene, M.; Li, X.; Knox, J. E.; Hratchian, H. P.; Cross, J. B.; Adamo, C.; Jaramillo, J.; Gomperts, R.; Stratmann, R. E.; Yazyev, O.; Austin, A. J.; Cammi, R.; Pomelli, C.; Ochterski, J. W.; Ayala, P. Y.; Morokuma, K.; Voth, G. A.; Salvador, P.; Dannenberg, J. J.; Zakrzewski, V. G.; Dapprich, S.; Daniels, A. D.; Strain, M. C.; Farkas, O.; Malick, D. K.; Rabuck, A. D.; Raghavachari, K.; Foresman, J. B.; Ortiz, J. V.; Cui, Q.; Baboul, A. G.; Clifford, S.; Cioslowski, J.; Stefanov, B. B.; Liu, G.; Liashenko, A.; Piskorz, P.; Komaromi, I.; Martin, R. L.; Fox, D. J.; Keith, T.; Al-Laham, M. A.; Peng, C. Y.; Nanayakkara, A.; Challacombe, M.; Gill, P. M. W.; Johnson, B.; Chen, W.; Wong, M. W.; Gonzalez, C.; and Pople, J. A., Gaussian, Inc.,Wallingford CT, 2004. 


\section{Geometry Summaries}

Cartesian coordinates and distances are reported in Ångstroms. Electronic energies are reported in Hartrees.

2 (9,10,11,29,21,22-Hexaphenyltetrabenzo[a,c,l,n]pentacene) - Singlet

RB3LYP/6-31G(d) optimized structure:

\begin{tabular}{|c|c|c|c|}
\hline C & 4.869077 & $\odot .596038$ & 1.311359 \\
\hline C & 3.612076 & $\odot .495729$ & $\odot .534100$ \\
\hline C & 6.089665 & ๑.198222 & $\odot .707170$ \\
\hline C & 3.612103 & $-\odot .495602$ & -0.534117 \\
\hline C & 4.869129 & $-\odot .595892$ & -1.311335 \\
\hline C & 6.089692 & $-\odot .198067$ & -0.707103 \\
\hline C & 2.432477 & 1.192111 & 0.782742 \\
\hline C & 1.202612 & ๑. 696018 & 0.193852 \\
\hline C & 2.432532 & -1.192018 & -0.782794 \\
\hline C & 1.202635 & -0.695956 & -0.193947 \\
\hline C & $-\odot .0 \odot \odot \odot 27$ & 1.427048 & $-0.00 \odot \odot 57$ \\
\hline C & -1.202644 & $\odot .695975$ & -0.193952 \\
\hline C & 0.000021 & -1.427025 & -0.000036 \\
\hline C & -1.202619 & $-\odot .695993$ & 0.193872 \\
\hline C & -2.432483 & -1.192071 & 0.782774 \\
\hline C & -3.612080 & -0.495687 & 0.534122 \\
\hline C & -2.432537 & 1.192020 & -0.782819 \\
\hline C & -3.612111 & $\odot .495613$ & -0.534122 \\
\hline C & -4.869138 & $\odot .595870$ & -1.311346 \\
\hline C & -6.089697 & $\odot .198034$ & -0.707112 \\
\hline C & -4.869080 & $-\odot .595987$ & 1.311382 \\
\hline C & -6.089672 & $-\odot .198217$ & 0.707172 \\
\hline C & 7.267307 & -0.178416 & -1.475540 \\
\hline C & 7.262723 & -0.536824 & -2.816334 \\
\hline $\mathrm{H}$ & 8.193715 & 0.158633 & -1.021888 \\
\hline $\mathrm{H}$ & 8.184317 & -0.505249 & -3.391323 \\
\hline C & 4.884210 & -0.925808 & -2.682413 \\
\hline C & 6.058680 & -0.904832 & -3.423158 \\
\hline $\mathrm{H}$ & 3.958917 & -1.175073 & -3.182968 \\
\hline $\mathrm{H}$ & 6.029840 & -1.156058 & -4.479992 \\
\hline C & 4.884111 & ๑.925954 & 2.682439 \\
\hline C & 6.058555 & $\odot .904991$ & 3.423223 \\
\hline $\mathrm{H}$ & 3.958799 & 1.175213 & 3.182964 \\
\hline $\mathrm{H}$ & 6.029678 & 1.156218 & 4.480056 \\
\hline C & 7.267253 & 0.178578 & 1.475648 \\
\hline C & 7.262621 & $\odot .536989$ & 2.816441 \\
\hline $\mathrm{H}$ & 8.193677 & -0.158469 & 1.022030 \\
\hline $\mathrm{H}$ & 8.184196 & 0.505418 & 3.391462 \\
\hline C & -4.884221 & ๑. 925758 & -2.682432 \\
\hline C & -6.058686 & $\odot .9 \odot 4743$ & -3.423182 \\
\hline $\mathrm{H}$ & -3.958931 & 1.175035 & -3.182988 \\
\hline $\mathrm{H}$ & -6.029847 & 1.155948 & -4.480022 \\
\hline C & -7.267306 & ๑.178337 & -1.475556 \\
\hline C & -7.262723 & 0.536717 & -2.816358 \\
\hline $\mathrm{H}$ & -8.193708 & -0.158727 & -1.021905 \\
\hline
\end{tabular}




\begin{tabular}{|c|c|c|c|}
\hline $\mathrm{H}$ & -8.184313 & $\odot .505106$ & -3.391352 \\
\hline$C$ & -4.884113 & -0.925858 & 2.682471 \\
\hline C & -6.058562 & -0.904895 & 3.423249 \\
\hline $\mathrm{H}$ & -3.958798 & -1.175075 & 3.183010 \\
\hline $\mathrm{H}$ & -6.029684 & -1.156084 & 4.480091 \\
\hline C & -7.267266 & -0.178578 & 1.475642 \\
\hline C & -7.262633 & -0.536944 & 2.816448 \\
\hline $\mathrm{H}$ & -8.193696 & 0.158430 & 1.022008 \\
\hline $\mathrm{H}$ & -8.184212 & -0.505378 & 3. 391462 \\
\hline C & 2.389503 & 2.335700 & 1.743348 \\
\hline C & 3. 223481 & 3.452603 & 1.584777 \\
\hline C & 1.556832 & 2.279822 & 2.872902 \\
\hline C & 3.234376 & 4.475558 & 2.530789 \\
\hline $\mathrm{H}$ & 3.868347 & 3.511702 & 0.713261 \\
\hline C & 1.574001 & 3.297848 & 3.825928 \\
\hline $\mathrm{H}$ & ๑.905265 & 1.420853 & 3.008835 \\
\hline C & 2.415122 & 4.399452 & 3.659292 \\
\hline $\mathrm{H}$ & 3.885772 & 5.333736 & 2.387476 \\
\hline $\mathrm{H}$ & ๑.930359 & 3.229483 & 4.699314 \\
\hline $\mathrm{H}$ & 2.429111 & 5.194358 & 4.400300 \\
\hline C & $-\odot .0 \odot \odot \odot 5 \odot$ & 2. 917677 & $-0.0 \odot \odot \odot 44$ \\
\hline C & 0.848014 & 3.636938 & -0.856277 \\
\hline C & -0.848130 & 3.636895 & 0.856209 \\
\hline C & 0.843442 & 5.030343 & -0.862188 \\
\hline $\mathrm{H}$ & 1.503919 & 3.094820 & -1.531427 \\
\hline C & -0.843593 & 5.030300 & 0.862156 \\
\hline $\mathrm{H}$ & -1.504020 & 3.094743 & 1.531348 \\
\hline C & $-\odot .000 \odot 84$ & 5.733005 & -0.000007 \\
\hline $\mathrm{H}$ & 1.499392 & 5.568088 & -1.541993 \\
\hline $\mathrm{H}$ & -1.499556 & 5.568011 & 1.541975 \\
\hline $\mathrm{H}$ & -๑.๑०७०९८ & 6.819880 & $\odot .0 \odot \odot \odot \odot 7$ \\
\hline C & -2.389636 & 2.335595 & -1.743445 \\
\hline C & -1.557008 & 2.279730 & -2.873031 \\
\hline C & -3.223657 & 3.452465 & -1.584864 \\
\hline C & -1.574258 & 3.297737 & -3.826076 \\
\hline $\mathrm{H}$ & $-\odot .905411$ & 1.420785 & -3.008974 \\
\hline C & -3.234631 & 4.475402 & -2.530894 \\
\hline $\mathrm{H}$ & -3.868493 & 3.511553 & -0.713326 \\
\hline C & -2.415417 & 4.399309 & -3.659428 \\
\hline $\mathrm{H}$ & $-\odot .930648$ & 3. 229382 & -4.699487 \\
\hline $\mathrm{H}$ & -3.886058 & 5.333555 & -2.387573 \\
\hline $\mathrm{H}$ & -2.429469 & 5.194200 & -4.400452 \\
\hline C & -2.389527 & -2.335647 & 1.743397 \\
\hline C & -3.223581 & -3.452501 & 1.584882 \\
\hline C & -1.556805 & -2.279799 & 2.872915 \\
\hline C & -3.234502 & -4.475437 & 2.530914 \\
\hline $\mathrm{H}$ & -3.868492 & -3.511572 & 0.713398 \\
\hline C & -1.573992 & -3.297811 & 3.825956 \\
\hline $\mathrm{H}$ & -0.905179 & -1.420867 & 3.008807 \\
\hline C & -2.415190 & -4.399364 & 3.659377 \\
\hline $\mathrm{H}$ & -3.885960 & -5.333576 & 2.387645 \\
\hline $\mathrm{H}$ & -0.930306 & -3.229472 & 4.699312 \\
\hline $\mathrm{H}$ & -2.429195 & -5.194256 & 4.400400 \\
\hline C & ๑.0๑००55 & -2.917653 & $-0.0 \odot \odot \odot 26$ \\
\hline C & $-\odot .848045$ & -3.636921 & -0.856218 \\
\hline C & 0.848204 & -3.636864 & 0.856163 \\
\hline $\mathrm{C}$ & $-\odot .843451$ & -5.030325 & $-\odot .862141$ \\
\hline
\end{tabular}




$\begin{array}{rrrr}\text { H } & -1.503999 & -3.094806 & -1.531324 \\ \mathrm{C} & 0.843700 & -5.030269 & 0.862090 \\ \mathrm{H} & 1.504128 & -3.094706 & 1.531264 \\ \mathrm{C} & 0.000146 & -5.732980 & -0.000024 \\ \mathrm{H} & -1.499434 & -5.568075 & -1.541910 \\ \mathrm{H} & 1.499720 & -5.567975 & 1.541858 \\ \mathrm{H} & 0.000182 & -6.819855 & -0.000023 \\ \mathrm{C} & 2.389646 & -2.335643 & -1.743361 \\ \mathrm{C} & 3.223682 & -3.452492 & -1.584713 \\ \mathrm{C} & 1.557020 & -2.279854 & -2.872953 \\ \mathrm{C} & 3.234679 & -4.475481 & -2.530687 \\ \mathrm{H} & 3.868516 & -3.511519 & -0.713169 \\ \mathrm{C} & 1.574290 & -3.297915 & -3.825940 \\ \mathrm{H} & 0.905411 & -1.420927 & -3.008949 \\ \mathrm{C} & 2.415468 & -4.399464 & -3.659227 \\ \mathrm{H} & 3.886120 & -5.333615 & -2.387315 \\ \mathrm{H} & 0.930683 & -3.229620 & -4.699357 \\ \mathrm{H} & 2.429535 & -5.194395 & -4.400207 \\ & & & \\ \text { E(RB+HF- LYP })= & -2847.59595584 & \end{array}$


2 (9,10,11,29,21,22-Hexaphenyltetrabenzo[a,c,l,n]pentacene) - Triplet

\section{UB3LYP/6-31G(d) optimized structure:}

\begin{tabular}{|c|c|c|c|}
\hline C & 4.884098 & $-\odot .595954$ & -1.300192 \\
\hline & 3.637060 & $-\odot .457992$ & -0.543642 \\
\hline C & 6.116578 & $-\odot .211759$ & -0.697162 \\
\hline C & 3.636959 & 0.458132 & 0.543904 \\
\hline C & 4.883875 & $\odot .596158$ & 1.300639 \\
\hline C & 6.116462 & ๑. 211975 & $\odot .697811$ \\
\hline C & 2.409665 & -1.156480 & -0.829631 \\
\hline C & 1.213848 & $-\odot .688505$ & -0.234593 \\
\hline C & 2.409475 & 1.156582 & 0.829673 \\
\hline C & 1.213780 & $\odot .688510$ & $\odot .234518$ \\
\hline C & $\odot .000061$ & -1.429224 & $-\odot .000119$ \\
\hline C & -1.213768 & $-\odot .688567$ & 0.234490 \\
\hline C & $-0.000 \odot 45$ & 1.429167 & $-\odot . \odot \odot \odot 11 \odot$ \\
\hline C & -1.213823 & $\odot .688450$ & -0.234616 \\
\hline C & -2.409633 & 1.156450 & -0.829649 \\
\hline C & -3.637036 & $\odot .457972$ & -0.543685 \\
\hline C & -2.409479 & -1.156635 & ๑.829624 \\
\hline C & -3.636955 & $-\odot .458182$ & 0.543835 \\
\hline C & -4.883876 & $-\odot .596215$ & 1.300555 \\
\hline C & -6.116455 & -0.211996 & 0.697734 \\
\hline C & -4.884067 & 0.595976 & -1.300236 \\
\hline C & -6.116557 & 0.211783 & $-\odot .697226$ \\
\hline C & 7.298375 & $\odot .239390$ & 1.466280 \\
\hline C & 7.288023 & $\odot .619590$ & 2.797204 \\
\hline $\mathrm{H}$ & 8.232292 & $-\odot .082393$ & 1.017640 \\
\hline $\mathrm{H}$ & 8.211080 & 0.620340 & 3.370609 \\
\hline C & 4.899529 & $\odot .953060$ & 2.671762 \\
\hline C & 6.073213 & $\odot .967079$ & 3.405110 \\
\hline $\mathrm{H}$ & 3.970499 & 1.184801 & 3.173512 \\
\hline $\mathrm{H}$ & 6.043326 & 1.229764 & 4.459112 \\
\hline C & 4.899966 & $-\odot .952818$ & -2.671320 \\
\hline C & 6.073764 & $-\odot .966800$ & $-3.4 \odot 4487$ \\
\hline $\mathrm{H}$ & 3.971013 & -1.184564 & -3.173215 \\
\hline $\mathrm{H}$ & 6.044044 & -1.229457 & -4.458500 \\
\hline C & 7.298614 & -0.239144 & -1.465446 \\
\hline C & 7.288475 & -0.619314 & -2.796380 \\
\hline $\mathrm{H}$ & 8.232456 & ๑.082642 & -1.016653 \\
\hline $\mathrm{H}$ & 8.211621 & -0.620041 & -3.369640 \\
\hline C & -4.899537 & $-\odot .953170$ & 2.671664 \\
\hline C & -6.073223 & $-\odot .967199$ & 3.405008 \\
\hline $\mathrm{H}$ & -3.970510 & -1.184950 & 3.173404 \\
\hline $\mathrm{H}$ & -6.043343 & -1.229926 & 4.459000 \\
\hline C & -7.298370 & -0.239420 & 1.466200 \\
\hline C & -7.288027 & $-\odot .619668$ & 2.797111 \\
\hline $\mathrm{H}$ & -8.232281 & ๑. 082394 & 1.017570 \\
\hline $\mathrm{H}$ & -8.211084 & -0.620425 & 3.370513 \\
\hline C & -4.899915 & 0.952889 & -2.671351 \\
\hline C & -6.073706 & 0.966913 & $-3.4 \odot 4528$ \\
\hline $\mathrm{H}$ & -3.970953 & 1.184641 & -3.173227 \\
\hline $\mathrm{H}$ & -6.043973 & 1.229607 & -4.458532 \\
\hline C & -7.298585 & ๑. 239211 & -1.465521 \\
\hline C & -7.288428 & 0.619424 & -2.796443 \\
\hline
\end{tabular}




\begin{tabular}{|c|c|c|c|}
\hline H & -8.232437 & $-\odot .082575$ & -1.016747 \\
\hline $\mathrm{H}$ & -8.211569 & 0.620185 & -3.369710 \\
\hline C & 2.402394 & -2.333574 & -1.743029 \\
\hline C & 3.288783 & -3.409448 & -1.567661 \\
\hline C & 1.542390 & -2.355671 & -2.854058 \\
\hline C & 3.319005 & -4.465602 & -2.474779 \\
\hline $\mathrm{H}$ & 3.958227 & -3.411958 & $-\odot .712905$ \\
\hline C & 1.579651 & -3.407397 & -3.769005 \\
\hline $\mathrm{H}$ & $\odot .854798$ & -1.528312 & -3.006270 \\
\hline C & 2.469838 & -4.466446 & -3.583973 \\
\hline $\mathrm{H}$ & 4.009383 & -5.290070 & -2.316834 \\
\hline $\mathrm{H}$ & ๑. 913412 & -3.397678 & -4.627867 \\
\hline $\mathrm{H}$ & 2.500429 & -5.286782 & -4.296151 \\
\hline C & $\odot .0 \odot \odot \odot 5 \odot$ & -2.914364 & $-0.0 \odot \odot 266$ \\
\hline C & $\odot .894609$ & -3.636350 & 0.807715 \\
\hline C & -0.894527 & -3.636165 & $-\odot .808391$ \\
\hline C & ๑.888365 & -5.029124 & ๑. 814821 \\
\hline $\mathrm{H}$ & 1.588502 & -3.095490 & 1.444908 \\
\hline C & -0.888318 & -5.028937 & -0.815781 \\
\hline $\mathrm{H}$ & -1.588413 & -3.095157 & -1.445470 \\
\hline C & ๑.0๑००15 & -5.732852 & -0.000552 \\
\hline $\mathrm{H}$ & 1.579930 & -5.566361 & 1.458871 \\
\hline $\mathrm{H}$ & -1.579897 & -5.566026 & -1.459940 \\
\hline $\mathrm{H}$ & ๑. .0०००००2 & -6.819638 & $-\odot .000662$ \\
\hline C & -2.402058 & -2.333807 & 1.742922 \\
\hline C & -1.541954 & -2.355948 & 2.853872 \\
\hline C & -3.288409 & -3.409711 & 1.567548 \\
\hline C & -1.579077 & -3.407750 & 3.768737 \\
\hline $\mathrm{H}$ & -0.854395 & -1.528562 & 3.006092 \\
\hline C & -3.318489 & -4.465943 & 2.474579 \\
\hline $\mathrm{H}$ & -3.957932 & -3.412184 & 0.712853 \\
\hline C & -2.469222 & -4.466833 & 3.583697 \\
\hline $\mathrm{H}$ & $-\odot .912764$ & -3.398064 & 4.627542 \\
\hline $\mathrm{H}$ & -4.008838 & -5.290434 & 2.316630 \\
\hline $\mathrm{H}$ & -2.499706 & -5.287229 & 4.295810 \\
\hline C & -2.402352 & 2.333590 & -1.742985 \\
\hline C & -3.288666 & 3.409508 & -1.567501 \\
\hline C & -1.542412 & 2.355704 & -2.854064 \\
\hline C & -3.318875 & 4.465721 & -2.474549 \\
\hline $\mathrm{H}$ & -3.958057 & 3.412008 & -0.712703 \\
\hline C & -1.579663 & 3.407488 & -3.768944 \\
\hline $\mathrm{H}$ & -0.854879 & 1.528312 & -3.006367 \\
\hline C & -2.469773 & 4.466581 & -3.583793 \\
\hline $\mathrm{H}$ & -4.009192 & 5.290222 & -2.316512 \\
\hline $\mathrm{H}$ & -0.913476 & 3.397780 & -4.627846 \\
\hline $\mathrm{H}$ & -2.500355 & 5.286964 & -4.295919 \\
\hline C & $-\odot .000 \odot 43$ & 2.914306 & $-\odot .00 \odot 238$ \\
\hline C & -0.894661 & 3.636274 & 0.807692 \\
\hline C & 0.894572 & 3.636124 & -0.808307 \\
\hline C & $-\odot .888439$ & 5.029048 & 0.814805 \\
\hline $\mathrm{H}$ & -1.588588 & 3.095400 & 1.444837 \\
\hline C & 0.888343 & 5.028897 & -0.815689 \\
\hline $\mathrm{H}$ & 1.588501 & 3.095131 & -1.445350 \\
\hline C & $-\odot .0 \odot \odot \odot 5 \odot$ & 5.732794 & $-\odot .0 \odot \odot 5 \odot 9$ \\
\hline $\mathrm{H}$ & -1.580053 & 5.566271 & 1.458815 \\
\hline $\mathrm{H}$ & 1.579952 & $5.56600 \odot$ & -1.459804 \\
\hline H & $-\odot .000053$ & 6.819580 & $-\odot .000613$ \\
\hline
\end{tabular}




$\begin{array}{llll}\text { C } & 2.402024 & 2.333787 & 1.742929 \\ \text { C } & 3.288404 & 3.409669 & 1.567568 \\ \text { C } & 1.541827 & 2.356001 & 2.853805 \\ \text { C } & 3.318437 & 4.465941 & 2.474555 \\ \text { H } & 3.957989 & 3.412092 & 0.712923 \\ \text { C } & 1.578899 & 3.407845 & 3.768624 \\ \text { H } & 0.854233 & 1.528641 & 3.006002 \\ \text { C } & 2.469084 & 4.466898 & 3.583607 \\ \text { H } & 4.008814 & 5.290411 & 2.316621 \\ \text { H } & 0.912515 & 3.398215 & 4.627374 \\ \text { H } & 2.499529 & 5.287327 & 4.295685 \\ \text { E } & & & \end{array}$


Tetrabenzo[a,c,l,n]pentacene $\left(0^{\circ}\right)$ - Singlet

\section{B3LYP/6-31G(d) optimized structure:}

\begin{tabular}{|c|c|c|c|}
\hline C & -4.967565 & -1.452303 & $-0.000 \odot \odot 1$ \\
\hline & -3.686108 & -0.725716 & $-0.000 \odot \odot 1$ \\
\hline C & -6.193851 & -0.737010 & $-\odot .0 \odot \odot \odot \odot 2$ \\
\hline C & -3.686109 & 0.725716 & $-0.000 \odot \odot 1$ \\
\hline C & -4.967563 & 1.452301 & $-0.000 \odot \odot 2$ \\
\hline C & -6.193851 & 0.737006 & -0.000001 \\
\hline C & -2.472488 & -1.390290 & $-0.000 \odot \odot 1$ \\
\hline C & -1.222253 & -0.721745 & $0.00000 \odot$ \\
\hline $\mathrm{H}$ & -2.440144 & -2.473926 & $-0.0 \odot \odot \odot \odot 1$ \\
\hline C & -2.472489 & 1.390290 & $-\odot .0 \odot \odot \odot \odot 1$ \\
\hline C & -1.222253 & 0.721746 & 0.000000 \\
\hline $\mathrm{H}$ & -2.440153 & 2.473927 & $-\odot .000 \odot \odot 1$ \\
\hline C & $\odot .0 \odot \odot \odot \odot \odot$ & -1.407285 & ๑. .๑०९०९ \\
\hline C & 1.222253 & -0.721746 & $\odot .0 \odot \odot \odot \odot \odot ~$ \\
\hline $\mathrm{H}$ & ๑.๑๑९९७९ & -2.495502 & $\odot . ๑ \odot \odot \odot \odot \odot ~$ \\
\hline C & $\odot .0 \odot \odot ० \odot \odot$ & 1.407285 & $\odot .000 \odot \odot \odot$ \\
\hline C & 1.222253 & 0.721745 & $\odot .0 \odot \odot \odot \odot 1$ \\
\hline $\mathrm{H}$ & $\odot .0 \odot \odot \odot \odot \odot$ & 2.495502 & $\odot .0 \odot \odot \odot \odot \odot$ \\
\hline C & 2.472488 & 1.390290 & $\odot .0 \odot \odot \odot \odot 1$ \\
\hline C & 3.686108 & 0.725716 & $\odot .00 \odot \odot \odot 2$ \\
\hline $\mathrm{H}$ & 2.440144 & 2.473926 & $\odot .0 \odot \odot \odot \odot 1$ \\
\hline C & 2.472489 & -1.390290 & $\odot .0 \odot \odot \odot \odot \odot$ \\
\hline C & 3.686109 & -0.725716 & $\odot .000001$ \\
\hline $\mathrm{H}$ & 2.440153 & -2.473927 & $\odot . ๑ \odot \odot \odot \odot \odot ~$ \\
\hline C & 4.967563 & -1.452301 & $\odot .0 \odot \odot \odot \odot 1$ \\
\hline C & 6.193851 & -0.737006 & $\odot . ๑ \odot \odot \odot \odot 2$ \\
\hline C & 4.967565 & 1.452303 & $\odot .0 \odot \odot \odot \odot 2$ \\
\hline C & 6.193851 & ๑.737010 & $\odot .0 \odot \odot \odot \odot 1$ \\
\hline C & -7.399084 & 1.470786 & $\odot .00 \odot \odot \odot 1$ \\
\hline C & -7.416244 & 2.855965 & $\odot .0 \odot \odot \odot \odot \odot$ \\
\hline $\mathrm{H}$ & -8.349485 & 0.951134 & $\odot .0000 \odot 3$ \\
\hline $\mathrm{H}$ & -8.364602 & 3.386149 & ๑. .๑०००2 \\
\hline C & -7.399076 & -1.470789 & $-\odot .0 \odot \odot \odot \odot 4$ \\
\hline C & -7.416237 & -2.855965 & -0.000004 \\
\hline $\mathrm{H}$ & -8.349479 & -0.951139 & -0.000006 \\
\hline $\mathrm{H}$ & -8.364594 & -3.386147 & $-0.0 \odot \odot \odot \odot 6$ \\
\hline C & -5.011769 & -2.862708 & $-0.000 \odot \odot 1$ \\
\hline C & -6.208107 & -3.560323 & - ๑. .००००2 \\
\hline $\mathrm{H}$ & -4.090156 & -3.432373 & 0.000001 \\
\hline $\mathrm{H}$ & -6.202013 & -4.646802 & $-\odot .0 \odot \odot \odot \odot 1$ \\
\hline C & -5.011765 & 2.862711 & $-\odot .0 \odot \odot \odot \odot 2$ \\
\hline C & -6.208103 & 3.560329 & 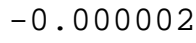 \\
\hline $\mathrm{H}$ & -4.090146 & 3.432367 & $-0.0 \odot \odot \odot \odot 4$ \\
\hline $\mathrm{H}$ & -6.202011 & 4.646810 & - ๑. .०००००2 \\
\hline C & 7.399076 & 1.470789 & $-\odot .00 \odot \odot \odot 1$ \\
\hline C & 7.416237 & 2.855965 & 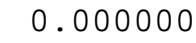 \\
\hline $\mathrm{H}$ & 8.349479 & 0.951139 & $-\odot . \odot \odot \odot \odot \odot ૩$ \\
\hline $\mathrm{H}$ & 8.364594 & 3.386147 & $-\odot . \odot \odot \odot \odot \odot 1$ \\
\hline C & 5.011769 & 2.862708 & 0.000004 \\
\hline C & 6.208107 & 3.560323 & ๑.๑०९००३ \\
\hline $\mathrm{H}$ & 4.090156 & 3.432373 & $\odot .00 \odot \odot \odot 6$ \\
\hline $\mathrm{H}$ & 6.202013 & 4.646802 & $\odot . \odot \odot \odot \odot \odot 4$ \\
\hline
\end{tabular}




\begin{tabular}{|c|c|c|c|}
\hline C & 7.399084 & -1.470786 & $\odot .0 \odot \odot \odot \odot 4$ \\
\hline C & 7.416244 & -2.855965 & $\odot .0 \odot \odot \odot \odot 3$ \\
\hline $\mathrm{H}$ & 8.349485 & $-\odot .951134$ & $\odot . \odot \odot \odot \odot \odot 6$ \\
\hline $\mathrm{H}$ & $8.3646 \odot 2$ & -3.386149 & $\odot . \odot \odot \odot \odot \odot 5$ \\
\hline C & 5.011765 & -2.862711 & $\odot .0 \odot \odot \odot \odot \odot$ \\
\hline C & 6.208103 & -3.560329 & $\odot .0 \odot \odot \odot \odot 1$ \\
\hline $\mathrm{H}$ & 4.090146 & -3.432367 & $-\odot . \odot \odot \odot \odot \odot 2$ \\
\hline $\mathrm{H}$ & 6.202011 & -4.646810 & $0.00000 \odot$ \\
\hline & LYP) $=$ & 1.38794 & \\
\hline
\end{tabular}


Tetrabenzo[a,c,l,n]pentacene $\left(0^{\circ}\right)$ - Triplet

\section{UB3LYP/6-31G(d) optimized structure:}

\begin{tabular}{|c|c|c|c|}
\hline C & 4.983205 & 1.440690 & $-\odot .00 \odot \odot \odot 2$ \\
\hline C & 3.723027 & $\odot .708326$ & $-\odot .0 \odot \odot \odot \odot 1$ \\
\hline C & 6.219883 & $\odot .731036$ & $-0.00 \odot \odot \odot 2$ \\
\hline C & 3.723027 & -0.708325 & $-\odot .0 \odot \odot \odot \odot 1$ \\
\hline C & $4.9832 \odot 4$ & -1.440690 & $-\odot .0 \odot \odot \odot \odot 1$ \\
\hline C & 6.219883 & -0.731036 & $-\odot .0 \odot \odot \odot \odot 1$ \\
\hline C & 2.470391 & 1.384558 & $-\odot .0 \odot \odot \odot \odot 1$ \\
\hline C & 1.244314 & $\odot .722086$ & $0.000 \odot \odot \odot$ \\
\hline $\mathrm{H}$ & 2.444530 & 2.467954 & $-\odot .0 \odot \odot \odot \odot 1$ \\
\hline C & 2.470391 & -1.384557 & $-\odot .0 \odot \odot \odot \odot 1$ \\
\hline C & 1.244314 & -0.722086 & ๑. 000000 \\
\hline $\mathrm{H}$ & 2.444531 & -2.467953 & $\odot . \odot \odot \odot \odot \odot \odot$ \\
\hline C & $-\odot . \odot \odot \odot \odot \odot 1$ & 1.408100 & $\odot . \odot \odot \odot \odot \odot \odot$ \\
\hline C & -1.244315 & $\odot .722086$ & $\odot .0 \odot \odot \odot \odot \odot$ \\
\hline H & $-\odot .000 \odot \odot 1$ & 2.495816 & $\odot ., \odot \odot \odot \odot \odot$ \\
\hline C & $-\odot . \odot \odot \odot \odot \odot 1$ & -1.408100 & $\odot . ๑ \odot \odot \odot \odot \odot$ \\
\hline C & -1.244315 & -0.722086 & $\odot . \odot \odot \odot \odot \odot \odot$ \\
\hline $\mathrm{H}$ & $\odot .00000 \odot$ & -2.495816 & $\odot . \odot \odot \odot \odot \odot \odot$ \\
\hline C & -2.470392 & -1.384558 & $\odot .0 \odot \odot \odot \odot 1$ \\
\hline C & -3.723027 & -0.708326 & $\odot .000001$ \\
\hline H & -2.444531 & -2.467953 & $\odot . \odot \odot \odot \odot \odot 1$ \\
\hline C & -2.470392 & 1.384557 & $\odot . \odot \odot \odot \odot \odot \odot$ \\
\hline C & -3.723027 & $\odot .708325$ & $\odot .0 \odot \odot \odot \odot 1$ \\
\hline $\mathrm{H}$ & -2.444532 & 2.467953 & $\odot .0 \odot \odot \odot \odot \odot$ \\
\hline C & -4.983204 & 1.440689 & $\odot .0 \odot \odot \odot \odot 1$ \\
\hline C & -6.219882 & 0.731036 & $\odot .00 \odot \odot \odot 1$ \\
\hline C & -4.983205 & -1.440689 & $\odot . \odot \odot \odot \odot \odot 2$ \\
\hline C & -6.219883 & -0.731035 & $\odot .0 \odot \odot \odot \odot 2$ \\
\hline C & 7.422155 & -1.474485 & $-\odot .0 \odot \odot \odot \odot 1$ \\
\hline C & 7.427996 & -2.856964 & $-\odot .0 \odot \odot \odot \odot 1$ \\
\hline $\mathrm{H}$ & 8.375428 & -0.959610 & $-\odot .0 \odot \odot \odot \odot 1$ \\
\hline $\mathrm{H}$ & 8.371718 & -3.395291 & $-\odot .0 \odot \odot \odot \odot 1$ \\
\hline C & 7.422156 & 1.474482 & $-\odot .00 \odot \odot \odot 2$ \\
\hline C & 7.427999 & 2.856962 & $-\odot .00 \odot \odot \odot 3$ \\
\hline $\mathrm{H}$ & 8.375429 & $\odot .959606$ & $-0.00 \odot \odot \odot 3$ \\
\hline $\mathrm{H}$ & 8.371723 & 3.395288 & $-0.000 \odot \odot 3$ \\
\hline C & 5.020501 & 2.856742 & $-\odot .00 \odot \odot \odot 2$ \\
\hline C & 6.211691 & 3.556128 & $-0.00 \odot \odot \odot 2$ \\
\hline $\mathrm{H}$ & 4.096250 & 3.421664 & $-\odot .0 \odot \odot \odot \odot 1$ \\
\hline H & 6.202352 & 4.642619 & $-\odot .0 \odot \odot \odot \odot 2$ \\
\hline C & 5.020498 & -2.856741 & $-\odot .0 \odot \odot \odot \odot 1$ \\
\hline C & 6.211686 & -3.556129 & $-\odot .0 \odot \odot \odot \odot 1$ \\
\hline $\mathrm{H}$ & 4.096245 & -3.421662 & -0.000001 \\
\hline $\mathrm{H}$ & 6.202347 & -4.642620 & $-\odot .0 \odot \odot \odot \odot 1$ \\
\hline C & -7.422156 & -1.474482 & ๑. $0 \odot \odot \odot \odot 1$ \\
\hline C & -7.427999 & -2.856961 & $\odot . \odot \odot \odot \odot \odot 2$ \\
\hline $\mathrm{H}$ & -8.375429 & -0.959606 & $\odot .0 \odot \odot \odot \odot 1$ \\
\hline $\mathrm{H}$ & -8.371722 & -3.395287 & $\odot .0 \odot \odot \odot \odot 2$ \\
\hline C & -5.020501 & -2.856741 & $\odot .0 \odot \odot \odot \odot 2$ \\
\hline C & -6.211690 & -3.556127 & $\odot . \odot \odot \odot \odot \odot 2$ \\
\hline $\mathrm{H}$ & -4.096250 & -3.421663 & $\odot . \odot \odot \odot \odot \odot 2$ \\
\hline H & -6.202352 & -4.642618 & $\odot .0 \odot \odot \odot \odot 2$ \\
\hline
\end{tabular}




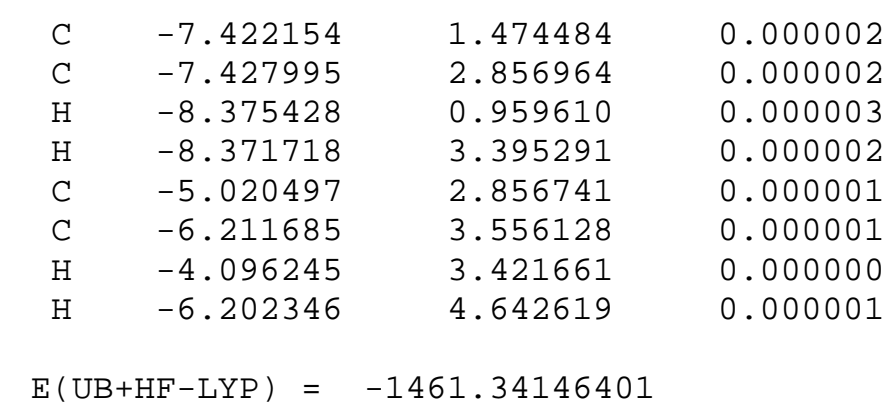


Tetrabenzo[a,c,l,n]pentacene $\left(149^{\circ}\right)$ - Singlet

\section{B3LYP/6-31G(d) optimized structure:}

\begin{tabular}{|c|c|c|c|}
\hline C & 4.860074 & -1.212485 & 0.762213 \\
\hline C & 3. 618859 & -0.449928 & $\odot .569812$ \\
\hline C & 6.063588 & -0.709160 & 0.196667 \\
\hline C & 3.619243 & 0.456687 & -0.564774 \\
\hline C & 4.863388 & 1.214868 & -0.760322 \\
\hline C & 6.067883 & $\odot .706209$ & -0.200014 \\
\hline C & 2.444398 & -0.595179 & 1.273659 \\
\hline C & 1.209201 & -0.141400 & $\odot .712233$ \\
\hline C & 2.443876 & 0.602218 & -1.267599 \\
\hline C & 1.208992 & 0.145939 & -0.706454 \\
\hline C & $\odot .0 \odot \odot \odot \odot 2$ & $-\odot .0 \odot \odot 4 \odot 7$ & 1.408520 \\
\hline C & -1.209220 & 0.140780 & 0.712222 \\
\hline C & -๑.๑๑७००८ & $-\odot .00 \odot 355$ & -1.402711 \\
\hline C & -1.208978 & -0.146503 & -0.706478 \\
\hline C & -2.444017 & -0.602622 & -1.267562 \\
\hline C & -3.619278 & $-\odot .456901$ & -0.564653 \\
\hline C & -2.444268 & 0.594697 & 1.273735 \\
\hline C & -3.618824 & $\odot .449691$ & $\odot .569958$ \\
\hline C & -4.859854 & 1.212428 & 0.762391 \\
\hline C & -6.063471 & $\odot .709457$ & 0.196650 \\
\hline C & -4.863610 & -1.214905 & -0.760176 \\
\hline C & -6.067994 & $-\odot .705909$ & -0.200033 \\
\hline C & 7.222369 & 1.500046 & -0.222299 \\
\hline C & 7.201242 & 2.773686 & -0.785545 \\
\hline $\mathrm{H}$ & 8.140318 & 1.119324 & 0.216283 \\
\hline $\mathrm{H}$ & 8.100227 & 3.383986 & -0.776754 \\
\hline C & 4.865382 & 2.493884 & -1.338786 \\
\hline C & 6.024594 & 3.264126 & -1.360811 \\
\hline H & 3.938880 & 2.900427 & -1.734316 \\
\hline $\mathrm{H}$ & 6.005199 & 4.257514 & -1.800763 \\
\hline C & 4.858859 & -2.491542 & 1.340825 \\
\hline C & 6.014515 & -3.267027 & 1.358117 \\
\hline $\mathrm{H}$ & 3.932159 & -2.893958 & 1.740028 \\
\hline $\mathrm{H}$ & 5.992456 & -4.260234 & 1.798349 \\
\hline C & 7.214681 & -1.508518 & $\odot .214045$ \\
\hline C & 7.190766 & -2.781921 & 0.777465 \\
\hline $\mathrm{H}$ & 8.132363 & -1.131936 & -0.228658 \\
\hline $\mathrm{H}$ & 8.087077 & -3.396077 & 0.764700 \\
\hline C & -4.858394 & 2.491428 & 1.341122 \\
\hline C & -6.013856 & 3. 267212 & 1.358350 \\
\hline $\mathrm{H}$ & -3.931629 & 2.893581 & 1.740444 \\
\hline $\mathrm{H}$ & -5.991616 & 4.260371 & 1.798682 \\
\hline C & -7.214313 & 1.509137 & 0.213923 \\
\hline C & -7.190142 & 2.782485 & 0.777472 \\
\hline $\mathrm{H}$ & -8.132024 & 1.132816 & -0.228948 \\
\hline $\mathrm{H}$ & -8.086256 & 3.396922 & 0.764590 \\
\hline C & -4.865850 & -2.493968 & -1.338532 \\
\hline C & -6.025252 & -3.263920 & -1.360613 \\
\hline $\mathrm{H}$ & -3.939418 & -2.900762 & -1.733961 \\
\hline $\mathrm{H}$ & $-6.00604 \odot$ & -4.257351 & -1.800475 \\
\hline C & -7.222726 & -1.499424 & -0.222425 \\
\hline C & -7.201857 & -2.773112 & -0.785559 \\
\hline
\end{tabular}




$\begin{array}{rrrr}H & -8.140641 & -1.118437 & 0.215994 \\ H & -8.101033 & -3.383137 & -0.776881 \\ H & -2.427747 & 1.112223 & 2.229904 \\ H & -2.427229 & -1.119729 & -2.223973 \\ H & 0.000033 & -0.000336 & -2.490616 \\ H & -0.000036 & -0.000418 & 2.496417 \\ H & 2.428021 & -1.112794 & 2.229778 \\ H & 2.426950 & 1.119276 & -2.224037 \\ \text { E } & & \end{array}$


Tetrabenzo[a,c,l,n]pentacene $\left(149^{\circ}\right)$ - Triplet

\section{UB3LYP/6-31G(d) optimized structure:}

\begin{tabular}{|c|c|c|c|}
\hline C & 4.878159 & -1.212457 & 0.743612 \\
\hline C & 3.646618 & -0.473874 & $\odot .526246$ \\
\hline C & 6.095251 & -0.703186 & ๑.195291 \\
\hline C & 3.646840 & 0.479977 & -0.521446 \\
\hline C & 4.880891 & 1.214842 & -0.741894 \\
\hline C & 6.099062 & $\odot .701079$ & -0.198341 \\
\hline C & 2.425816 & -0.660462 & 1.231468 \\
\hline C & 1.221881 & -0.183670 & ๑.705688 \\
\hline C & 2.424992 & 0.666333 & -1.225644 \\
\hline C & 1.221500 & 0.186661 & -0.700269 \\
\hline C & 0.000133 & $-\odot .000845$ & 1.411426 \\
\hline C & -1.221764 & 0.182080 & 0.705872 \\
\hline C & -0.000136 & $-\odot .00 \odot 835$ & -1.405722 \\
\hline C & -1.221621 & -0.188242 & -0.700088 \\
\hline C & -2.425381 & -0.667464 & -1.225363 \\
\hline C & -3.647062 & -0.480647 & -0.521060 \\
\hline C & -2.425433 & 0.659315 & 1.231758 \\
\hline C & -3.646401 & 0.473199 & $\odot .526637$ \\
\hline C & -4.877546 & 1.212445 & 0.743908 \\
\hline C & -6.094856 & ๑.703915 & 0.195374 \\
\hline C & -4.881506 & -1.214847 & -0.741609 \\
\hline C & -6.099452 & -0.700348 & -0.198259 \\
\hline C & 7.259066 & 1.492045 & -0.256451 \\
\hline C & 7.232496 & 2.757795 & -0.827607 \\
\hline $\mathrm{H}$ & 8.183938 & 1.111997 & 0.167623 \\
\hline $\mathrm{H}$ & 8.133758 & 3.364538 & -0.841968 \\
\hline C & 4.885744 & 2.489544 & -1.345346 \\
\hline C & 6.044194 & 3.250880 & -1.388799 \\
\hline $\mathrm{H}$ & 3.957018 & 2.895905 & -1.735596 \\
\hline $\mathrm{H}$ & 6.024876 & 4.240094 & -1.838156 \\
\hline C & 4.880425 & -2.487412 & 1.346811 \\
\hline C & 6.035896 & -3.253309 & 1.385688 \\
\hline $\mathrm{H}$ & 3.951560 & -2.890266 & 1.740281 \\
\hline $\mathrm{H}$ & 6.014460 & -4.242510 & 1.834975 \\
\hline C & 7.252467 & -1.499020 & 0.248628 \\
\hline C & 7.223764 & -2.764695 & $\odot .819628$ \\
\hline $\mathrm{H}$ & 8.177021 & -1.122442 & -0.179227 \\
\hline $\mathrm{H}$ & 8.122810 & -3.374797 & 0.830122 \\
\hline C & -4.879190 & 2.487331 & 1.347268 \\
\hline C & -6.034217 & 3.253901 & 1.385991 \\
\hline $\mathrm{H}$ & -3.950172 & 2.889596 & 1.740978 \\
\hline $\mathrm{H}$ & -6.012286 & 4.243040 & 1.835391 \\
\hline C & -7.251615 & 1.500424 & ๑. 248562 \\
\hline C & -7.222268 & 2.766041 & 0.819658 \\
\hline $\mathrm{H}$ & -8.176311 & 1.124415 & -0.179484 \\
\hline $\mathrm{H}$ & -8.120955 & 3.376675 & 0.830041 \\
\hline C & -4.886982 & -2.489612 & -1.344908 \\
\hline C & -6.045873 & -3.250274 & -1.388515 \\
\hline $\mathrm{H}$ & -3.958414 & -2.896559 & -1.734925 \\
\hline $\mathrm{H}$ & -6.027051 & -4.239545 & -1.837767 \\
\hline C & -7.259909 & -1.490637 & -0.256517 \\
\hline C & -7.233984 & -2.756439 & -0.827586 \\
\hline
\end{tabular}




$\begin{array}{rrrr}H & -8.184634 & -1.110022 & 0.167373 \\ H & -8.135603 & -3.362650 & -0.842058 \\ H & -2.436025 & 1.135258 & 2.208990 \\ H & -2.435729 & -1.143568 & -2.202540 \\ H & -0.000196 & -0.001028 & -2.493002 \\ H & 0.000195 & -0.001046 & 2.498694 \\ H & 2.436666 & -1.136571 & 2.208614 \\ H & 2.435082 & 1.142284 & -2.202899 \\ & & \end{array}$


Table S1. Absolute energies for anthracene as a function of twist angle (B3LYP/6-31G(d)).

\begin{tabular}{|c|c|c|c|c|c|c|c|}
\hline \multirow{2}{*}{$\begin{array}{c}\text { Angle } \\
\text { (Degrees) }\end{array}$} & \multicolumn{3}{|c|}{$(\mathrm{au})$} & \multicolumn{2}{|c|}{$\mathrm{S}_{0}$} & \multicolumn{2}{|c|}{$\mathrm{T}_{1}$} \\
\hline & $\mathrm{S}_{0 \text { (closed-shell) }}$ & $\mathrm{S}_{0 \text { (open-shell) }}$ & $\mathrm{T}_{1}$ & $\left\langle S^{2}\right\rangle$ & $\left\langle\mathrm{S}^{2}\right\rangle_{\text {annihilation }}$ & $\left\langle S^{2}\right\rangle$ & $\left\langle\mathrm{S}^{2}\right\rangle_{\text {annihilation }}$ \\
\hline 0.0 & -539.5305235 & -539.5305235 & -539.4639468 & 0.0000 & 0.0000 & 2.0213 & 2.0003 \\
\hline 10.0 & -539.5297302 & -539.5297302 & -539.4632527 & 0.0000 & 0.0000 & 2.0214 & 2.0003 \\
\hline 20.0 & -539.5273363 & -539.5273363 & -539.4611575 & 0.0000 & 0.0000 & 2.0216 & 2.0003 \\
\hline 30.0 & -539.5233214 & -539.5233214 & -539.4576399 & 0.0000 & 0.0000 & 2.0219 & 2.0003 \\
\hline 40.0 & -539.5176853 & -539.5176853 & -539.4526920 & 0.0000 & 0.0000 & 2.0223 & 2.0003 \\
\hline 50.0 & -539.5104015 & -539.5104015 & -539.4462692 & 0.0000 & 0.0000 & 2.0229 & 2.0003 \\
\hline 60.0 & -539.5013875 & -539.5013875 & -539.4382641 & 0.0000 & 0.0000 & 2.0237 & 2.0003 \\
\hline 70.0 & -539.4905684 & -539.4905684 & -539.4285844 & 0.0000 & 0.0000 & 2.0246 & 2.0004 \\
\hline 80.0 & -539.4779297 & -539.4779297 & -539.4172157 & 0.0000 & 0.0000 & 2.0257 & 2.0004 \\
\hline 90.0 & -539.4634609 & -539.4634609 & -539.4041371 & 0.0000 & 0.0000 & 2.0270 & 2.0004 \\
\hline 100.0 & -539.4470989 & -539.4470989 & -539.3892768 & 0.0000 & 0.0000 & 2.0287 & 2.0005 \\
\hline 110.0 & -539.4288097 & -539.4288097 & -539.3725827 & 0.0000 & 0.0000 & 2.0306 & 2.0006 \\
\hline 120.0 & -539.4085698 & -539.4085698 & -539.3540419 & 0.0000 & 0.0000 & 2.0329 & 2.0007 \\
\hline 130.0 & -539.3863364 & -539.3863364 & -539.3345236 & 0.0000 & 0.0000 & 2.0596 & 2.0020 \\
\hline 140.0 & -539.3620653 & -539.3620653 & -539.3141730 & 0.0000 & 0.0000 & 2.0767 & 2.0034 \\
\hline 150.0 & -539.3360347 & -539.3360347 & -539.2932740 & 0.0000 & 0.0000 & 2.0849 & 2.0045 \\
\hline 160.0 & -539.3091795 & -539.3091795 & -539.2720726 & 0.0000 & 0.0000 & 2.0846 & 2.0045 \\
\hline 170.0 & -539.2814323 & -539.2814323 & -539.2506805 & 0.0000 & 0.0000 & 2.0829 & 2.0043 \\
\hline 180.0 & -539.2526004 & -539.2526004 & -539.2293724 & 0.0000 & 0.0000 & 2.0836 & 2.0042 \\
\hline
\end{tabular}

Table S2. Absolute energies for tetracene as a function of twist angle (B3LYP/6-31G(d)).

\begin{tabular}{|c|c|c|c|c|c|c|c|}
\hline \multirow{2}{*}{$\begin{array}{c}\text { Angle } \\
\text { (Degrees) }\end{array}$} & \multicolumn{3}{|c|}{$(\mathrm{au})$} & \multicolumn{2}{|c|}{$\mathrm{S}_{0}$} & \multicolumn{2}{|c|}{$\mathrm{T}_{1}$} \\
\hline & $\mathrm{S}_{0 \text { (closed-shell) }}$ & $\mathrm{S}_{0 \text { (open-shell) }}$ & $\mathrm{T}_{1}$ & $\left\langle\mathrm{~S}^{2}\right\rangle$ & $\left\langle\mathrm{S}^{2}\right\rangle_{\text {annihilation }}$ & $\left\langle\mathrm{S}^{2}\right\rangle$ & $\left\langle\mathrm{S}^{2}\right\rangle_{\text {annihilation }}$ \\
\hline 0.0 & -693.1658124 & -693.1658124 & -693.1216627 & 0.0000 & 0.0000 & 2.0275 & 2.0004 \\
\hline 10.0 & -693.1652388 & -693.1652388 & -693.1211368 & 0.0000 & 0.0000 & 2.0275 & 2.0004 \\
\hline 20.0 & -693.1635081 & -693.1635081 & -693.1195499 & 0.0000 & 0.0000 & 2.0276 & 2.0004 \\
\hline 30.0 & -693.1606136 & -693.1606136 & -693.1168961 & 0.0000 & 0.0000 & 2.0279 & 2.0004 \\
\hline 40.0 & -693.1565760 & -693.1565761 & -693.1131919 & 0.0000 & 0.0000 & 2.0282 & 2.0005 \\
\hline 50.0 & -693.1513872 & -693.1513872 & -693.1084171 & 0.0000 & 0.0000 & 2.0286 & 2.0005 \\
\hline 60.0 & -693.1449833 & -693.1449833 & -693.1024924 & 0.0000 & 0.0000 & 2.0291 & 2.0005 \\
\hline 70.0 & -693.1373260 & -693.1373260 & -693.0953775 & 0.0000 & 0.0000 & 2.0298 & 2.0005 \\
\hline 80.0 & -693.1284446 & -693.1284446 & -693.0871045 & 0.0000 & 0.0000 & 2.0305 & 2.0005 \\
\hline 90.0 & -693.1183426 & -693.1183426 & -693.0776624 & 0.0000 & 0.0000 & 2.0315 & 2.0006 \\
\hline 100.0 & -693.1069693 & -693.1069693 & -693.0669796 & 0.0000 & 0.0000 & 2.0325 & 2.0006 \\
\hline 110.0 & -693.0943061 & -693.0943061 & -693.0550232 & 0.0000 & 0.0000 & 2.0338 & 2.0007 \\
\hline 120.0 & -693.0803444 & -693.0803443 & -693.0417889 & 0.0000 & 0.0000 & 2.0352 & 2.0352 \\
\hline 130.0 & -693.0650636 & -693.0650636 & -693.0272548 & 0.0000 & 0.0000 & 2.0368 & 2.0008 \\
\hline 140.0 & -693.0484381 & -693.0484381 & -693.0113931 & 0.0000 & 0.0000 & 2.0387 & 2.0009 \\
\hline 150.0 & -693.0304444 & -693.0304444 & -692.9941882 & 0.0000 & 0.0000 & 2.0409 & 2.0010 \\
\hline 160.0 & -693.0110491 & -693.0110491 & -692.9756195 & 0.0000 & 0.0000 & 2.0435 & 2.0011 \\
\hline 170.0 & -692.9902206 & -692.9902206 & -692.9556522 & 0.0000 & 0.0000 & 2.0464 & 2.0012 \\
\hline 180.0 & -692.9680602 & -692.9680602 & -692.9342694 & 0.0000 & 0.0000 & 2.0497 & 2.0014 \\
\hline
\end{tabular}


Table S3. Absolute energies for pentacene as a function of twist angle (B3LYP/6-31G(d)).

\begin{tabular}{|c|c|c|c|c|c|c|c|}
\hline \multirow{2}{*}{$\begin{array}{c}\text { Angle } \\
\text { (Degrees) }\end{array}$} & \multicolumn{3}{|c|}{$(\mathrm{au})$} & \multicolumn{2}{|c|}{$\mathrm{S}_{0}$} & \multicolumn{2}{|c|}{$\mathrm{T}_{1}$} \\
\hline & $\mathrm{S}_{0 \text { (closed-shell) }}$ & $\mathrm{S}_{0 \text { (open-shell) }}$ & $\mathrm{T}_{1}$ & $\left\langle S^{2}\right\rangle$ & $\left\langle\mathrm{S}^{2}\right\rangle_{\text {annihilation }}$ & $\left\langle S^{2}\right\rangle$ & $\left\langle\mathrm{S}^{2}\right\rangle_{\text {annihilation }}$ \\
\hline 0.0 & -846.7999434 & -846.7999434 & -846.7714549 & 0.0000 & 0.0000 & 2.0307 & 2.0006 \\
\hline 10.0 & -846.7994947 & -846.7994947 & -846.7710315 & 0.0000 & 0.0000 & 2.0307 & 2.0006 \\
\hline 20.0 & -846.7981403 & -846.7981403 & -846.7697538 & 0.0000 & 0.0000 & 2.0308 & 2.0006 \\
\hline 30.0 & -846.7958781 & -846.7958781 & -846.7676215 & 0.0000 & 0.0000 & 2.0310 & 2.0006 \\
\hline 40.0 & -846.7927371 & -846.7927371 & -846.7646632 & 0.0000 & 0.0000 & 2.0313 & 2.0006 \\
\hline 50.0 & -846.7887168 & -846.7887168 & -846.7608715 & 0.0000 & 0.0000 & 2.0316 & 2.0006 \\
\hline 60.0 & -846.7837643 & -846.7837643 & -846.7561838 & 0.0000 & 0.0000 & 2.0320 & 2.0006 \\
\hline 70.0 & -846.7778619 & -846.7778619 & -846.7505830 & 0.0000 & 0.0000 & 2.0325 & 2.0007 \\
\hline 80.0 & -846.7710497 & -846.7710497 & -846.7441074 & 0.0000 & 0.0000 & 2.0332 & 2.0007 \\
\hline 90.0 & -846.7633218 & -846.7633218 & -846.7367347 & 0.0000 & 0.0000 & 2.0339 & 2.0007 \\
\hline 100.0 & -846.7546207 & -846.7546207 & -846.7283947 & 0.0000 & 0.0000 & 2.0347 & 2.0007 \\
\hline 110.0 & -846.7449400 & -846.7449400 & -846.7188617 & 0.0000 & 0.0000 & 2.0347 & 2.0007 \\
\hline 120.0 & -846.7342974 & -846.7342974 & -846.7088091 & 0.0000 & 0.0000 & 2.0367 & 2.0008 \\
\hline 130.0 & -846.7226860 & -846.7226860 & -846.6975752 & 0.0000 & 0.0000 & 2.0379 & 2.0009 \\
\hline 140.0 & -846.7100872 & -846.7100872 & -846.6853478 & 0.0000 & 0.0000 & 2.0392 & 2.0009 \\
\hline 150.0 & -846.6964936 & -846.6964936 & -846.6721186 & 0.0000 & 0.0000 & 2.0408 & 2.0010 \\
\hline 160.0 & -846.6818884 & -846.6818884 & -846.6578682 & 0.0000 & 0.0000 & 2.0426 & 2.0011 \\
\hline 170.0 & -846.6662466 & -846.6662466 & -846.6425676 & 0.0000 & 0.0000 & 2.0446 & 2.0012 \\
\hline 180.0 & -846.6495472 & -846.6495472 & -846.6261939 & 0.0000 & 0.0000 & 2.0469 & 2.0013 \\
\hline
\end{tabular}

Table S4. Absolute energies for hexacene as a function of twist angle (B3LYP/6-31G(d)).

\begin{tabular}{|c|c|c|c|c|c|c|}
\hline \multirow{2}{*}{$\begin{array}{c}\text { Angle } \\
\text { (Degrees) }\end{array}$} & \multicolumn{2}{|r|}{ (au) } & \multicolumn{2}{|c|}{$\mathrm{S}_{0}$} & \multicolumn{2}{|c|}{$\mathrm{T}_{1}$} \\
\hline & $\mathrm{S}_{0 \text { (closed-shell) }}$ & $\mathrm{S}_{0 \text { (open-shell) }}$ & $\left\langle\mathrm{S}^{2}\right\rangle$ & $\left\langle\mathrm{S}^{2}\right\rangle_{\text {annihilation }}$ & $\left\langle S^{2}\right\rangle$ & $\left\langle\mathrm{S}^{2}\right\rangle_{\text {annihilataion }}$ \\
\hline 0.0 & -1000.4334888 & $-1000.4336819-1000.4162565$ & 0.2602 & 0.0413 & 2.0343 & 2.0007 \\
\hline 10.0 & -1000.4331196 & $-1000.4333129-1000.4159023$ & 0.2588 & 0.0408 & 2.0344 & 2.0007 \\
\hline 20.0 & -1000.4320049 & $-1000.4321985-1000.4148332$ & 0.2560 & 0.0399 & 2.0345 & 2.0007 \\
\hline 30.0 & -1000.4301450 & $-1000.4303408-1000.4130519$ & 0.2615 & 0.0419 & 2.0346 & 2.0007 \\
\hline 40.0 & -1000.4275735 & $-1000.4277720-1000.4105934$ & 0.2642 & 0.0430 & 2.0348 & 2.0008 \\
\hline 50.0 & -1000.4242928 & $-1000.4244944-1000.4074553$ & 0.2682 & 0.0446 & 2.0351 & 2.0008 \\
\hline 60.0 & -1000.4202537 & $-1000.4204577-1000.4035797$ & 0.2713 & 0.0459 & 2.0355 & 2.0008 \\
\hline 70.0 & -1000.4154459 & $-1000.4156511-1000.3989556$ & 0.2733 & 0.0469 & 2.0359 & 2.0008 \\
\hline 80.0 & -1000.4099079 & $-1000.4101132-1000.3936191$ & 0.2759 & 0.0482 & 2.0364 & 2.0008 \\
\hline 90.0 & -1000.4036261 & $-1000.4038286-1000.3875492$ & 0.2771 & 0.0491 & 2.0369 & 2.0008 \\
\hline 100.0 & -1000.3965498 & $-1000.3967462-1000.3806968$ & 0.2767 & 0.0493 & 2.0376 & 2.0009 \\
\hline 110.0 & -1000.3886970 & $-1000.3888822-1000.3730805$ & 0.2749 & 0.0491 & 2.0383 & 2.0009 \\
\hline 120.0 & -1000.3800968 & $-1000.3802689-1000.3647239$ & 0.2718 & 0.0484 & 2.0391 & 2.0010 \\
\hline 130.0 & -1000.3707292 & $-1000.3708826-1000.3555954$ & 0.2614 & 0.0451 & 2.0401 & 2.0010 \\
\hline 140.0 & -1000.3605742 & $-1000.3607050-1000.3456724$ & 0.2563 & 0.0438 & 2.0411 & 2.0011 \\
\hline 150.0 & -1000.3496412 & $-1000.3497422-1000.3349651$ & 0.2332 & 0.0363 & 2.0423 & 2.0011 \\
\hline 160.0 & -1000.3379241 & $-1000.3379903-1000.3234554$ & 0.2000 & 0.0266 & 2.0436 & 2.0012 \\
\hline 170.0 & -1000.3253864 & $-1000.3254172-1000.3110995$ & 0.1390 & 0.0126 & 2.0451 & 2.0013 \\
\hline 180.0 & -1000.3120165 & $-1000.3120185-1000.2978867$ & 0.0259 & 0.0004 & 2.0467 & 2.0014 \\
\hline
\end{tabular}


Table S5. Absolute energies for heptacene as a function of twist angle (B3LYP/6-31G(d)).

\begin{tabular}{|c|c|c|c|c|c|}
\hline \multirow{2}{*}{$\begin{array}{c}\text { Angle } \\
\text { (Degrees) }\end{array}$} & $(\mathrm{au})$ & \multicolumn{2}{|c|}{$\mathrm{S}_{0}$} & \multicolumn{2}{|c|}{$\mathrm{T}_{1}$} \\
\hline & $\mathrm{S}_{0}$ (closed-shell) & $\left\langle\mathrm{S}^{2}\right\rangle$ & $\left\langle\mathrm{S}^{2}\right\rangle_{\text {annihilation }}$ & $\left\langle\mathrm{S}^{2}\right\rangle$ & $\left\langle\mathrm{S}^{2}\right\rangle_{\text {annihilation }}$ \\
\hline 0.0 & $-1154.0667232-1154.0694260-1154.0577715$ & 0.7967 & 0.6106 & 2.0376 & 2.0009 \\
\hline 10.0 & $-1154.0664087-1154.0691128-1154.0574668$ & 0.7955 & 0.6082 & 2.0376 & 2.0009 \\
\hline 20.0 & $-1154.0654590-1154.0681674-1154.0565469$ & 0.7967 & 0.6107 & 2.0377 & 2.0009 \\
\hline 30.0 & $-1154.0638762-1154.0665929-1154.0550164$ & 0.8003 & 0.6186 & 2.0378 & 2.0009 \\
\hline 40.0 & $-1154.0616970-1154.0644267-1154.0529143$ & 0.8018 & 0.6219 & 2.0380 & 2.0009 \\
\hline 50.0 & $-1154.0589245-1154.0616709-1154.0502403$ & 0.8057 & 0.6305 & 2.0383 & 2.0009 \\
\hline 60.0 & $-1154.0555103-1154.0582736-1154.0469380$ & 0.8118 & 0.644 & 2.0386 & 2.0009 \\
\hline 70.0 & $-1154.0514472-1154.0542268-1154.0429999$ & 0.8167 & 0.6553 & 2.0389 & 2.0010 \\
\hline 80.0 & $-1154.0467738-1154.0495689-1154.0384655$ & 0.8203 & 0.6642 & 2.0393 & 2.0010 \\
\hline 90.0 & $-1154.0414798-1154.0442912-1154.0333251$ & 0.8283 & 0.6828 & 2.0398 & 2.0010 \\
\hline 100.0 & $-1154.0355308-1154.0383567-1154.0275435$ & 0.8353 & 0.6995 & 2.0403 & 2.0010 \\
\hline 110.0 & $-1154.0289459-1154.0317844-1154.0211348$ & 0.8425 & 0.7174 & 2.0410 & 2.0011 \\
\hline 120.0 & $-1154.0217420-1154.0245833-1154.0140999$ & 0.8496 & 0.7358 & 2.0416 & 2.0011 \\
\hline 130.0 & $-1154.0138849-1154.0167175-1154.0064040$ & 0.8566 & 0.7547 & 2.0424 & 2.0011 \\
\hline 140.0 & $-1154.0053670-1154.0081798-1153.9980456$ & 0.8647 & 0.7768 & 2.0432 & 2.0012 \\
\hline 150.0 & $-1153.9962076-1153.9989898-1153.9890444$ & 0.8721 & 0.7982 & 2.0442 & 2.0012 \\
\hline 160.0 & $-1153.9863898-1153.9891254-1153.9793710$ & 0.8775 & 0.8158 & 2.0452 & 2.0013 \\
\hline 170.0 & $-1153.9758799-1153.9785480-1153.9689906$ & 0.8838 & 0.8363 & 2.0464 & 2.0014 \\
\hline 180.0 & $-1153.9646925-1153.9672733-1153.9579221$ & 0.8903 & 0.8581 & 2.0477 & 2.0014 \\
\hline
\end{tabular}

Table S6. Absolute energies for 2 and tetrabenzo[a,c,l,n]pentacene (B3LYP/6-31G(d)).

\begin{tabular}{|c|c|c|c|c|c|c|c|c|}
\hline & Angle & & $(\mathrm{au})$ & & & $\mathrm{S}_{0}$ & & $\mathrm{~T}_{1}$ \\
\hline & (Degrees) & $\mathrm{S}_{0 \text { (closed-shell) }}$ & $\mathrm{S}_{0 \text { (open-shell) }}$ & $\mathrm{T}_{1}$ & $\left\langle\mathrm{~S}^{2}\right\rangle$ & $\left\langle\mathrm{S}^{2}\right\rangle_{\text {annihilation }}$ & $\left\langle S^{2}\right\rangle$ & $\left\langle\mathrm{S}^{2}\right\rangle_{\text {annihilation }}$ \\
\hline 2 & - & -2847.5959558 & -2847.5959560 & -2847.5486926 & 0.0000 & 0.0000 & 2.0292 & 2.0005 \\
\hline tetrabenzo[a,c,l,n]pentacene & 0.0 & -1461.3879441 & - & -1461.3414640 & - & - & 2.0250 & 2.0004 \\
\hline tetrabenzo[a,c,l,n]pentacene & 149.0 & -1461.3226004 & - & -1461.2703002 & - & - & 2.0281 & 2.0005 \\
\hline Pentacene & 144.0 & -846.7047696 & - & -846.6801771 & - & - & 2.0399 & 2.0010 \\
\hline Pentacene & 149.0 & -846.6986032 & - & -846.6741737 & - & - & 2.0406 & 2.0010 \\
\hline
\end{tabular}


Table S7. HOMO and LUMO energies for anthracene (B3LYP/6-31G(d)).

\begin{tabular}{|c|c|c|c|c|c|c|}
\hline \multirow{2}{*}{$\begin{array}{c}\text { Angle } \\
\text { (Degrees) }\end{array}$} & \multicolumn{2}{|c|}{$(\mathrm{au})$} & \multirow[b]{2}{*}{ HOMO-LUMO } & \multicolumn{3}{|c|}{$(\mathrm{eV})$} \\
\hline & HOMO & LUMO & & $\mathrm{HOMO}$ & LUMO & HOMO-LUMO \\
\hline 0.0 & -0.19204 & -0.05998 & 0.13206 & -5.23 & -1.63 & 3.59 \\
\hline 10.0 & -0.19199 & -0.06003 & 0.13196 & -5.22 & -1.63 & 3.59 \\
\hline 20.0 & -0.19185 & -0.06017 & 0.13168 & -5.22 & -1.64 & 3.58 \\
\hline 30.0 & -0.19162 & -0.06040 & 0.13122 & -5.21 & -1.64 & 3.57 \\
\hline 40.0 & -0.19129 & -0.06071 & 0.13058 & -5.21 & -1.65 & 3.55 \\
\hline 50.0 & -0.19086 & -0.06106 & 0.12980 & -5.19 & -1.66 & 3.53 \\
\hline 60.0 & -0.19035 & -0.06143 & 0.12892 & -5.18 & -1.67 & 3.51 \\
\hline 70.0 & -0.18977 & -0.06179 & 0.12798 & -5.16 & -1.68 & 3.48 \\
\hline 80.0 & -0.18912 & -0.06211 & 0.12701 & -5.15 & -1.69 & 3.46 \\
\hline 90.0 & -0.18826 & -0.06250 & 0.12576 & -5.12 & -1.70 & 3.42 \\
\hline 100.0 & -0.18742 & -0.06273 & 0.12469 & -5.10 & -1.71 & 3.39 \\
\hline 110.0 & -0.18646 & -0.06288 & 0.12358 & -5.07 & -1.71 & 3.36 \\
\hline 120.0 & -0.18549 & -0.06291 & 0.12258 & -5.05 & -1.71 & 3.34 \\
\hline 130.0 & -0.18446 & -0.06284 & 0.12162 & -5.02 & -1.71 & 3.31 \\
\hline 140.0 & -0.18334 & -0.06266 & 0.12068 & -4.99 & -1.71 & 3.28 \\
\hline 150.0 & -0.18579 & -0.06053 & 0.12526 & -5.06 & -1.65 & 3.41 \\
\hline 160.0 & -0.18991 & -0.05763 & 0.13228 & -5.17 & -1.57 & 3.60 \\
\hline 170.0 & -0.19416 & -0.05470 & 0.13946 & -5.28 & -1.49 & 3.79 \\
\hline 180.0 & -0.19136 & -0.05192 & 0.13944 & -5.21 & -1.41 & 3.79 \\
\hline
\end{tabular}

Table S8. HOMO and LUMO energies for tetracene (B3LYP/6-31G(d)).

\begin{tabular}{|c|c|c|c|c|c|c|}
\hline \multirow{2}{*}{$\begin{array}{c}\text { Angle } \\
\text { (Degrees) }\end{array}$} & \multicolumn{2}{|c|}{$(\mathrm{au})$} & \multirow[b]{2}{*}{ HOMO-LUMO } & \multicolumn{3}{|c|}{$(\mathrm{eV})$} \\
\hline & HOMO & LUMO & & HOMO & LUMO & HOMO-LUMO \\
\hline 0.0 & -0.17845 & -0.07633 & 0.10212 & -4.86 & -2.08 & 2.78 \\
\hline 10.0 & -0.17844 & -0.07635 & 0.10209 & -4.86 & -2.08 & 2.78 \\
\hline 20.0 & -0.17839 & -0.07643 & 0.10196 & -4.85 & -2.08 & 2.77 \\
\hline 30.0 & -0.17832 & -0.07654 & 0.10178 & -4.85 & -2.08 & 2.77 \\
\hline 40.0 & -0.17822 & -0.07671 & 0.10151 & -4.85 & -2.09 & 2.76 \\
\hline 50.0 & -0.17809 & -0.07690 & 0.10119 & -4.85 & -2.09 & 2.75 \\
\hline 60.0 & -0.17794 & -0.07710 & 0.10084 & -4.84 & -2.10 & 2.74 \\
\hline 70.0 & -0.17778 & -0.07730 & 0.10048 & -4.84 & -2.10 & 2.73 \\
\hline 80.0 & -0.17759 & -0.07751 & 0.10008 & -4.83 & -2.11 & 2.72 \\
\hline 90.0 & -0.17739 & -0.07769 & 0.09970 & -4.83 & -2.11 & 2.71 \\
\hline 100.0 & -0.17719 & -0.07784 & 0.09935 & -4.82 & -2.12 & 2.70 \\
\hline 110.0 & -0.17686 & -0.07801 & 0.09885 & -4.81 & -2.12 & 2.69 \\
\hline 120.0 & -0.17664 & -0.07806 & 0.09858 & -4.81 & -2.12 & 2.68 \\
\hline 130.0 & -0.17637 & -0.07805 & 0.09832 & -4.80 & -2.12 & 2.68 \\
\hline 140.0 & -0.17615 & -0.07793 & 0.09822 & -4.79 & -2.12 & 2.67 \\
\hline 150.0 & -0.17596 & -0.07772 & 0.09824 & -4.79 & -2.11 & 2.67 \\
\hline 160.0 & -0.17578 & -0.07739 & 0.09839 & -4.78 & -2.11 & 2.68 \\
\hline 170.0 & -0.17572 & -0.07694 & 0.09878 & -4.78 & -2.09 & 2.69 \\
\hline 180.0 & -0.17790 & -0.07525 & 0.10265 & -4.84 & -2.05 & 2.79 \\
\hline
\end{tabular}


Table S9. HOMO and LUMO energies for pentacene (B3LYP/6-31G(d)).

\begin{tabular}{|c|c|c|c|c|c|c|}
\hline \multirow{2}{*}{$\begin{array}{c}\text { Angle } \\
\text { (Degrees) }\end{array}$} & \multicolumn{2}{|c|}{$(\mathrm{au})$} & \multirow[b]{2}{*}{ HOMO-LUMO } & \multicolumn{3}{|c|}{$(\mathrm{eV})$} \\
\hline & HOMO & LUMO & & HOMO & LUMO & HOMO-LUMO \\
\hline 0.0 & -0.16899 & -0.08772 & 0.08127 & -4.60 & -2.39 & 2.21 \\
\hline 10.0 & -0.16898 & -0.08774 & 0.08124 & -4.60 & -2.39 & 2.21 \\
\hline 20.0 & -0.16897 & -0.08778 & 0.08119 & -4.60 & -2.39 & 2.21 \\
\hline 30.0 & -0.16895 & -0.08784 & 0.08111 & -4.60 & -2.39 & 2.21 \\
\hline 40.0 & -0.16893 & -0.08794 & 0.08099 & -4.60 & -2.39 & 2.20 \\
\hline 50.0 & -0.16890 & -0.08805 & 0.08085 & -4.60 & -2.40 & 2.20 \\
\hline 60.0 & -0.16887 & -0.08818 & 0.08069 & -4.60 & -2.40 & 2.20 \\
\hline 70.0 & -0.16883 & -0.08831 & 0.08052 & -4.59 & -2.40 & 2.19 \\
\hline 80.0 & -0.16880 & -0.08845 & 0.08035 & -4.59 & -2.41 & 2.19 \\
\hline 90.0 & -0.16876 & -0.08858 & 0.08018 & -4.59 & -2.41 & 2.18 \\
\hline 100.0 & -0.16873 & -0.08868 & 0.08005 & -4.59 & -2.41 & 2.18 \\
\hline 110.0 & -0.16864 & -0.08879 & 0.07985 & -4.59 & -2.42 & 2.17 \\
\hline 120.0 & -0.16863 & -0.08885 & 0.07978 & -4.59 & -2.42 & 2.17 \\
\hline 130.0 & -0.16864 & -0.08886 & 0.07978 & -4.59 & -2.42 & 2.17 \\
\hline 140.0 & -0.16868 & -0.08883 & 0.07985 & -4.59 & -2.42 & 2.17 \\
\hline 150.0 & -0.16868 & -0.08876 & 0.07992 & -4.59 & -2.42 & 2.17 \\
\hline 160.0 & -0.16877 & -0.08860 & 0.08017 & -4.59 & -2.41 & 2.18 \\
\hline 170.0 & -0.16891 & -0.08835 & 0.08056 & -4.60 & -2.40 & 2.19 \\
\hline 180.0 & -0.16910 & -0.08801 & 0.08109 & -4.60 & -2.39 & 2.21 \\
\hline
\end{tabular}

Table S10. HOMO and LUMO energies for hexacene (B3LYP/6-31G(d)).

\begin{tabular}{|c|c|c|c|c|c|c|}
\hline \multirow{2}{*}{$\begin{array}{c}\text { Angle } \\
\text { (Degrees) }\end{array}$} & \multicolumn{2}{|c|}{$(\mathrm{au})$} & \multirow[b]{2}{*}{ HOMO-LUMO } & \multicolumn{3}{|c|}{$(\mathrm{eV})$} \\
\hline & HOMO & LUMO & & HOMO & LUMO & HOMO-LUMO \\
\hline 0.0 & -0.16382 & -0.09425 & 0.06957 & -4.46 & -2.56 & 1.89 \\
\hline 10.0 & -0.16382 & -0.09426 & 0.06956 & -4.46 & -2.56 & 1.89 \\
\hline 20.0 & -0.16382 & -0.09433 & 0.06949 & -4.46 & -2.57 & 1.89 \\
\hline 30.0 & -0.16382 & -0.09433 & 0.06949 & -4.46 & -2.57 & 1.89 \\
\hline 40.0 & -0.16382 & -0.09439 & 0.06943 & -4.46 & -2.57 & 1.89 \\
\hline 50.0 & -0.16383 & -0.09446 & 0.06937 & -4.46 & -2.57 & 1.89 \\
\hline 60.0 & -0.16383 & -0.09455 & 0.06928 & -4.46 & -2.57 & 1.89 \\
\hline 70.0 & -0.16382 & -0.09466 & 0.06916 & -4.46 & -2.58 & 1.88 \\
\hline 80.0 & -0.16381 & -0.09477 & 0.06904 & -4.46 & -2.58 & 1.88 \\
\hline 90.0 & -0.16379 & -0.09489 & 0.06890 & -4.46 & -2.58 & 1.87 \\
\hline 100.0 & -0.16377 & -0.09502 & 0.06875 & -4.46 & -2.59 & 1.87 \\
\hline 110.0 & -0.16375 & -0.09516 & 0.06859 & -4.46 & -2.59 & 1.87 \\
\hline 120.0 & -0.16371 & -0.09531 & 0.06840 & -4.45 & -2.59 & 1.86 \\
\hline 130.0 & -0.16365 & -0.09546 & 0.06819 & -4.45 & -2.60 & 1.86 \\
\hline 140.0 & -0.16357 & -0.09560 & 0.06797 & -4.45 & -2.60 & 1.85 \\
\hline 150.0 & -0.16348 & -0.09577 & 0.06771 & -4.45 & -2.61 & 1.84 \\
\hline 160.0 & -0.16337 & -0.09595 & 0.06742 & -4.45 & -2.61 & 1.83 \\
\hline 170.0 & -0.16324 & -0.09615 & 0.06709 & -4.44 & -2.62 & 1.83 \\
\hline 180.0 & -0.16308 & -0.09641 & 0.06667 & -4.44 & -2.62 & 1.81 \\
\hline
\end{tabular}


Table S11. HOMO and LUMO energies for heptacene (B3LYP/6-31G(d)).

\begin{tabular}{|c|c|c|c|c|c|c|}
\hline \multirow{2}{*}{$\begin{array}{c}\text { Angle } \\
\text { (Degrees) }\end{array}$} & \multicolumn{2}{|c|}{$(\mathrm{au})$} & \multirow[b]{2}{*}{ HOMO-LUMO } & \multicolumn{3}{|c|}{$(\mathrm{eV})$} \\
\hline & HOMO & LUMO & & HOMO & LUMO & HOMO-LUMO \\
\hline 0.0 & -0.16370 & -0.09552 & 0.06818 & -4.45 & -2.60 & 1.86 \\
\hline 10.0 & -0.16370 & -0.09556 & 0.06814 & -4.45 & -2.60 & 1.85 \\
\hline 20.0 & -0.16370 & -0.09558 & 0.06812 & -4.45 & -2.60 & 1.85 \\
\hline 30.0 & -0.16371 & -0.09559 & 0.06812 & -4.45 & -2.60 & 1.85 \\
\hline 40.0 & -0.16371 & -0.09567 & 0.06804 & -4.45 & -2.60 & 1.85 \\
\hline 50.0 & -0.16372 & -0.09573 & 0.06799 & -4.46 & -2.60 & 1.85 \\
\hline 60.0 & -0.16377 & -0.09575 & 0.06802 & -4.46 & -2.61 & 1.85 \\
\hline 70.0 & -0.16375 & -0.09585 & 0.06790 & -4.46 & -2.61 & 1.85 \\
\hline 80.0 & -0.16375 & -0.09599 & 0.06776 & -4.46 & -2.61 & 1.84 \\
\hline 90.0 & -0.16377 & -0.09605 & 0.06772 & -4.46 & -2.61 & 1.84 \\
\hline 100.0 & -0.16378 & -0.09618 & 0.06760 & -4.46 & -2.62 & 1.84 \\
\hline 110.0 & -0.16379 & -0.09629 & 0.06750 & -4.46 & -2.62 & 1.84 \\
\hline 120.0 & -0.16379 & -0.09641 & 0.06738 & -4.46 & -2.62 & 1.83 \\
\hline 130.0 & -0.16378 & -0.09653 & 0.06725 & -4.46 & -2.63 & 1.83 \\
\hline 140.0 & -0.16376 & -0.09665 & 0.06711 & -4.46 & -2.63 & 1.83 \\
\hline 150.0 & -0.16373 & -0.09678 & 0.06695 & -4.46 & -2.63 & 1.82 \\
\hline 160.0 & -0.16367 & -0.09689 & 0.06678 & -4.45 & -2.64 & 1.82 \\
\hline 170.0 & -0.16360 & -0.09701 & 0.06659 & -4.45 & -2.64 & 1.81 \\
\hline 180.0 & -0.16353 & -0.09713 & 0.06640 & -4.45 & -2.64 & 1.81 \\
\hline
\end{tabular}

Table S12. HOMO and LUMO energies for 2 and tetrabenzo[a,c,l,n]pentacene (B3LYP/6-31G(d)).

\begin{tabular}{cccccccc}
\hline & Angle & \multicolumn{2}{c}{$(\mathrm{au})$} & & & $(\mathrm{eV})$ \\
\hline System & (Degrees) & HOMO & LUMO & HOMO-LUMO & HOMO & LUMO & HOMO-LUMO \\
\hline $\mathbf{2}$ & - & -0.17367 & -0.07835 & 0.09532 & -4.73 & -2.13 & 2.59 \\
Tetrabenzo[a,c,l,n]pentacene & 0.0 & -0.17899 & -0.0807 & 0.09829 & -4.87 & -2.20 & 2.67 \\
Tetrabenzo[a,c,l,n]pentacene & 149.0 & -0.18196 & -0.07640 & 0.10556 & -4.95 & -2.08 & 2.87 \\
\hline
\end{tabular}


Table S13. Vertical $\mathrm{S}_{0}-\mathrm{S}_{1}$ transition energies calculated using TDDFT for polyacenes (B3LYP/6-31G(d)).

\begin{tabular}{|c|c|c|c|c|}
\hline & & Experimental & TDDFT/6-31G(d) & \\
\hline$n^{a}$ & $\begin{array}{c}\text { Angle } \\
\text { (Degrees) }\end{array}$ & $\begin{array}{c}\mathrm{S}_{0}-\mathrm{S}_{1} \\
(\mathrm{eV})(\mathrm{nm})\end{array}$ & $\begin{array}{c}\mathrm{S}_{0}-\mathrm{S}_{1} \\
(\mathrm{eV})(\mathrm{nm})\end{array}$ & $f$ \\
\hline \multirow[t]{3}{*}{3} & 0.0 & 3.35 [370] & 3.28 [379] & 0.0583 \\
\hline & 90.0 & & 3.03 [409] & 0.0465 \\
\hline & 180.0 & & 3.03 [409] & 0.0162 \\
\hline \multirow[t]{3}{*}{4} & 0.0 & $2.61[475]$ & 2.49 [497] & 0.0496 \\
\hline & 90.0 & & $2.38[521]$ & 0.0423 \\
\hline & 180.0 & & 2.30 [539] & 0.0256 \\
\hline \multirow[t]{3}{*}{5} & 0.0 & 2.14 [582] & 1.94 [638] & 0.0414 \\
\hline & 90.0 & & 1.88 [658] & 0.0363 \\
\hline & 180.0 & & 1.81 [683] & 0.0250 \\
\hline \multirow[t]{3}{*}{6} & 0.0 & 1.79 [695] & 1.54 [803] & 0.0338 \\
\hline & 90.0 & & 1.51 [823] & 0.0303 \\
\hline & 180.0 & & $1.46[847]$ & 0.0220 \\
\hline \multirow[t]{3}{*}{7} & 0.0 & & $1.24[1001]$ & 0.0274 \\
\hline & 90.0 & & $1.21[1021]$ & 0.0249 \\
\hline & 180.0 & & $1.18[1048]$ & 0.0186 \\
\hline
\end{tabular}

$\overline{a_{n}}=$ number of benzene rings in the polyacene

Table S14. Vertical $\mathrm{S}_{0}-\mathrm{S}_{1}$ transition energies calculated using TDDFT for $\mathbf{2}$ and tetrabenzo[a,c,l,n]pentacene (B3LYP/6-31G(d)).

\begin{tabular}{cccc}
\hline & & TDDFT/6-31G(d) \\
\hline System & Angle & $\mathrm{S}_{0}-\mathrm{S}_{1}$ \\
$(\mathrm{eV})(\mathrm{nm})$ & $\mathrm{f}$ \\
\hline $\mathbf{2}$ & (Degrees) & $2.18[568]$ & 0.1223 \\
tetrabenzo[a,c,l,n]pentacene & - & $2.36[525]$ & 0.0133 \\
tetrabenzo[a,c,l,n]pentacene & 0.0 & $2.48[500]$ & 0.0199 \\
\hline
\end{tabular}


Table S15. Distortion energy per benzene and twist angle per benzene for anthracene (B3LYP/6-31G(d)).

\begin{tabular}{|c|c|c|c|c|c|c|c|}
\hline \multirow{2}{*}{$\begin{array}{c}\text { Angle } \\
\text { (Degrees) }\end{array}$} & \multirow{2}{*}{$\begin{array}{c}\text { Angle/Benzene } \\
\text { (Degrees) }\end{array}$} & \multicolumn{2}{|c|}{$(\mathrm{au})$} & \multicolumn{4}{|c|}{$(\mathrm{kcal} / \mathrm{mol})$} \\
\hline & & $\mathrm{S}_{0 \text { (closed-shell) }}$ & $\mathrm{S}_{0 \text { (open-shell) }}$ & $\Delta \mathrm{S}_{0}$ (closed-shell) & $\begin{array}{c}\Delta \mathrm{S}_{0 \text { (closed-shell) }} \\
\text { /Benzene }\end{array}$ & $\Delta \mathrm{S}_{0 \text { (open-shell) }}$ & $\begin{array}{c}\Delta \mathrm{S}_{0 \text { (open-shell) }} \\
\text { /Benzene }\end{array}$ \\
\hline 0.0 & 0.0 & -539.5305235 & -539.5305235 & 0.0 & 0.0 & 0.0 & 0.0 \\
\hline 10.0 & 3.3 & -539.5297302 & -539.5297302 & 0.5 & 0.2 & 0.5 & 0.2 \\
\hline 20.0 & 6.7 & -539.5273363 & -539.5273363 & 2.0 & 0.7 & 2.0 & 0.7 \\
\hline 30.0 & 10.0 & -539.5233214 & -539.5233214 & 4.5 & 1.5 & 4.5 & 1.5 \\
\hline 40.0 & 13.3 & -539.5176853 & -539.5176853 & 8.1 & 2.7 & 8.1 & 2.7 \\
\hline 50.0 & 16.7 & -539.5104015 & -539.5104015 & 12.6 & 4.2 & 12.6 & 4.2 \\
\hline 60.0 & 20.0 & -539.5013875 & -539.5013875 & 18.3 & 6.1 & 18.3 & 6.1 \\
\hline 70.0 & 23.3 & -539.4905684 & -539.4905684 & 25.1 & 8.4 & 25.1 & 8.4 \\
\hline 80.0 & 26.7 & -539.4779297 & -539.4779297 & 33.0 & 11.0 & 33.0 & 11.0 \\
\hline 90.0 & 30.0 & -539.4634609 & -539.4634609 & 42.1 & 14.0 & 42.1 & 14.0 \\
\hline 100.0 & 33.3 & -539.4470989 & -539.4470989 & 52.3 & 17.4 & 52.3 & 17.4 \\
\hline 110.0 & 36.7 & -539.4288097 & -539.4288097 & 63.8 & 21.3 & 63.8 & 21.3 \\
\hline 120.0 & 40.0 & -539.4085698 & -539.4085698 & 76.5 & 25.5 & 76.5 & 25.5 \\
\hline 130.0 & 43.3 & -539.3863364 & -539.3863364 & 90.5 & 30.2 & 90.5 & 30.2 \\
\hline 140.0 & 46.7 & -539.3620653 & -539.3620653 & 105.7 & 35.2 & 105.7 & 35.2 \\
\hline 150.0 & 50.0 & -539.3360347 & -539.3360347 & 122.0 & 40.7 & 122.0 & 40.7 \\
\hline 160.0 & 53.3 & -539.3091795 & -539.3091795 & 138.9 & 46.3 & 138.9 & 46.3 \\
\hline 170.0 & 56.7 & -539.2814323 & -539.2814323 & 156.3 & 52.1 & 156.3 & 52.1 \\
\hline 180.0 & 60.0 & -539.2526004 & -539.2526004 & 174.4 & 58.1 & 174.4 & 58.1 \\
\hline
\end{tabular}

Table S16. Distortion energy per benzene and twist angle per benzene for tetracene (B3LYP/6-31G(d)).

\begin{tabular}{|c|c|c|c|c|c|c|c|}
\hline \multirow{2}{*}{$\begin{array}{c}\text { Angle } \\
\text { (Degrees) }\end{array}$} & \multirow{2}{*}{$\begin{array}{c}\text { Angle/Benzene } \\
\text { (Degrees) }\end{array}$} & \multicolumn{2}{|c|}{$(\mathrm{au})$} & \multicolumn{4}{|c|}{$(\mathrm{kcal} / \mathrm{mol})$} \\
\hline & & $\mathrm{S}_{0 \text { (closed-shell) }}$ & $\mathrm{S}_{0}$ (open-shell) & $\Delta \mathrm{S}_{0}$ (closed-shell) & $\begin{array}{l}\Delta \mathrm{S}_{0 \text { (closed-shell) }} \\
\text { /Benzene }\end{array}$ & $\Delta \mathrm{S}_{0}$ (open-shell) & $\begin{array}{c}\Delta \mathrm{S}_{0} \text { (open-shell) } \\
\text { /Benzene }\end{array}$ \\
\hline 0.0 & 0.0 & -693.1658124 & -693.1658124 & 0.0 & 0.0 & 0.0 & 0.0 \\
\hline 10.0 & 2.5 & -693.1652388 & -693.1652388 & 0.4 & 0.1 & 0.4 & 0.1 \\
\hline 20.0 & 5.0 & -693.1635081 & -693.1635081 & 1.4 & 0.4 & 1.4 & 0.4 \\
\hline 30.0 & 7.5 & -693.1606136 & -693.1606136 & 3.3 & 0.8 & 3.3 & 0.8 \\
\hline 40.0 & 10.0 & -693.1565760 & -693.1565761 & 5.8 & 1.4 & 5.8 & 1.4 \\
\hline 50.0 & 12.5 & -693.1513872 & -693.1513872 & 9.1 & 2.3 & 9.1 & 2.3 \\
\hline 60.0 & 15.0 & -693.1449833 & -693.1449833 & 13.1 & 3.3 & 13.1 & 3.3 \\
\hline 70.0 & 17.5 & -693.1373260 & -693.1373260 & 17.9 & 4.5 & 17.9 & 4.5 \\
\hline 80.0 & 20.0 & -693.1284446 & -693.1284446 & 23.4 & 5.9 & 23.4 & 5.9 \\
\hline 90.0 & 22.5 & -693.1183426 & -693.1183426 & 29.8 & 7.4 & 29.8 & 7.4 \\
\hline 100.0 & 25.0 & -693.1069693 & -693.1069693 & 36.9 & 9.2 & 36.9 & 9.2 \\
\hline 110.0 & 27.5 & -693.0943061 & -693.0943061 & 44.9 & 11.2 & 44.9 & 11.2 \\
\hline 120.0 & 30.0 & -693.0803444 & -693.0803443 & 53.6 & 13.4 & 53.6 & 13.4 \\
\hline 130.0 & 32.5 & -693.0650636 & -693.0650636 & 63.2 & 15.8 & 63.2 & 15.8 \\
\hline 140.0 & 35.0 & -693.0484381 & -693.0484381 & 73.7 & 18.4 & 73.7 & 18.4 \\
\hline 150.0 & 37.5 & -693.0304444 & -693.0304444 & 84.9 & 21.2 & 84.9 & 21.2 \\
\hline 160.0 & 40.0 & -693.0110491 & -693.0110491 & 97.1 & 24.3 & 97.1 & 24.3 \\
\hline 170.0 & 42.5 & -692.9902206 & -692.9902206 & 110.2 & 27.5 & 110.2 & 27.5 \\
\hline 180.0 & 45.0 & -692.9680602 & -692.9680602 & 124.1 & 31.0 & 124.1 & 31.0 \\
\hline
\end{tabular}


Table S17. Distortion energy per benzene and twist angle per benzene for pentacene (B3LYP/6-31G(d)).

\begin{tabular}{|c|c|c|c|c|c|c|c|}
\hline \multirow{2}{*}{$\begin{array}{c}\text { Angle } \\
\text { (Degrees) } \\
\end{array}$} & \multirow{2}{*}{$\begin{array}{c}\text { Angle/Benzene } \\
\text { (Degrees) }\end{array}$} & \multicolumn{2}{|c|}{$(\mathrm{au})$} & \multicolumn{4}{|c|}{$(\mathrm{kcal} / \mathrm{mol})$} \\
\hline & & $\mathrm{S}_{0 \text { (closed-shell) }}$ & $\mathrm{S}_{0 \text { (open-shell) }}$ & $\Delta \mathrm{S}_{0 \text { (closed-shell) }}$ & $\begin{array}{c}\Delta \mathrm{S}_{0 \text { (closed-shell) }} \\
\text { /Benzene }\end{array}$ & $\Delta \mathrm{S}_{0 \text { (open-shell) }}$ & $\begin{array}{c}\Delta \mathrm{S}_{0} \text { (open-shell) } \\
\text { /Benzene }\end{array}$ \\
\hline 0.0 & 0.0 & -846.7999434 & -846.7999434 & 0.0 & 0.0 & 0.0 & 0.0 \\
\hline 10.0 & 2.0 & -846.7994947 & -846.7994947 & 0.3 & 0.1 & 0.3 & 0.1 \\
\hline 20.0 & 4.0 & -846.7981403 & -846.7981403 & 1.1 & 0.2 & 1.1 & 0.2 \\
\hline 30.0 & 6.0 & -846.7958781 & -846.7958781 & 2.6 & 0.5 & 2.6 & 0.5 \\
\hline 40.0 & 8.0 & -846.7927371 & -846.7927371 & 4.5 & 0.9 & 4.5 & 0.9 \\
\hline 50.0 & 10.0 & -846.7887168 & -846.7887168 & 7.0 & 1.4 & 7.0 & 1.4 \\
\hline 60.0 & 12.0 & -846.7837643 & -846.7837643 & 10.2 & 2.0 & 10.2 & 2.0 \\
\hline 70.0 & 14.0 & -846.7778619 & -846.7778619 & 13.9 & 2.8 & 13.9 & 2.8 \\
\hline 80.0 & 16.0 & -846.7710497 & -846.7710497 & 18.1 & 3.6 & 18.1 & 3.6 \\
\hline 90.0 & 18.0 & -846.7633218 & -846.7633218 & 23.0 & 4.6 & 23.0 & 4.6 \\
\hline 100.0 & 20.0 & -846.7546207 & -846.7546207 & 28.4 & 5.7 & 28.4 & 5.7 \\
\hline 110.0 & 22.0 & -846.7449400 & -846.7449400 & 34.5 & 6.9 & 34.5 & 6.9 \\
\hline 120.0 & 24.0 & -846.7342974 & -846.7342974 & 41.2 & 8.2 & 41.2 & 8.2 \\
\hline 130.0 & 26.0 & -846.7226860 & -846.7226860 & 48.5 & 9.7 & 48.5 & 9.7 \\
\hline 140.0 & 28.0 & -846.7100872 & -846.7100872 & 56.4 & 11.3 & 56.4 & 11.3 \\
\hline 150.0 & 30.0 & -846.6964936 & -846.6964936 & 64.9 & 13.0 & 64.9 & 13.0 \\
\hline 160.0 & 32.0 & -846.6818884 & -846.6818884 & 74.1 & 14.8 & 74.1 & 14.8 \\
\hline 170.0 & 34.0 & -846.6662466 & -846.6662466 & 83.9 & 16.8 & 83.9 & 16.8 \\
\hline 180.0 & 36.0 & -846.6495472 & -846.6495472 & 94.4 & 18.9 & 94.4 & 18.9 \\
\hline
\end{tabular}

Table S18. Distortion energy per benzene and twist angle per benzene for hexacene (B3LYP/6-31G(d)).

\begin{tabular}{|c|c|c|c|c|c|c|}
\hline \multirow{2}{*}{$\begin{array}{c}\text { Angle } \\
\text { (Degrees) }\end{array}$} & \multirow{2}{*}{$\begin{array}{c}\text { Angle/Benzene } \\
\text { (Degrees) }\end{array}$} & $(\mathrm{au})$ & \multicolumn{4}{|c|}{$(\mathrm{kcal} / \mathrm{mol})$} \\
\hline & & $\mathrm{S}_{0}$ (closed-shell) & $\Delta \mathrm{S}_{0 \text { (closed-shell) }}$ & $\begin{array}{c}\Delta \mathrm{S}_{0 \text { (closed-shell) }} \\
\text { /Benzene }\end{array}$ & $\Delta \mathrm{S}_{0}$ (open-shell) & $\begin{array}{c}\Delta \mathrm{S}_{0 \text { (open-shell) }} \\
\text { /Benzene } \\
\end{array}$ \\
\hline 0.0 & 0.0 & $-1000.4334888-1000.4336819$ & 0.0 & 0.0 & 0.0 & 0.0 \\
\hline 10.0 & 1.7 & $-1000.4331196-1000.4333129$ & 0.2 & 0.0 & 0.2 & 0.0 \\
\hline 20.0 & 3.3 & $-1000.4320049-1000.4321985$ & 0.9 & 0.2 & 0.9 & 0.2 \\
\hline 30.0 & 5.0 & $-1000.4301450-1000.4303408$ & 2.1 & 0.3 & 2.1 & 0.3 \\
\hline 40.0 & 6.7 & $-1000.4275735-1000.4277720$ & 3.7 & 0.6 & 3.7 & 0.6 \\
\hline 50.0 & 8.3 & $-1000.4242928-1000.4244944$ & 5.8 & 1.0 & 5.8 & 1.0 \\
\hline 60.0 & 10.0 & $-1000.4202537-1000.4204577$ & 8.3 & 1.4 & 8.3 & 1.4 \\
\hline 70.0 & 11.7 & $-1000.4154459-1000.4156511$ & 11.3 & 1.9 & 11.3 & 1.9 \\
\hline 80.0 & 13.3 & $-1000.4099079-1000.4101132$ & 14.8 & 2.5 & 14.8 & 2.5 \\
\hline 90.0 & 15.0 & $-1000.4036261-1000.4038286$ & 18.7 & 3.1 & 18.7 & 3.1 \\
\hline 100.0 & 16.7 & $-1000.3965498-1000.3967462$ & 23.2 & 3.9 & 23.2 & 3.9 \\
\hline 110.0 & 18.3 & $-1000.3886970-1000.3888822$ & 28.1 & 4.7 & 28.1 & 4.7 \\
\hline 120.0 & 20.0 & $-1000.3800968-1000.3802689$ & 33.5 & 5.6 & 33.5 & 5.6 \\
\hline 130.0 & 21.7 & $-1000.3707292-1000.3708826$ & 39.4 & 6.6 & 39.4 & 6.6 \\
\hline 140.0 & 23.3 & $-1000.3605742-1000.3607050$ & 45.8 & 7.6 & 45.8 & 7.6 \\
\hline 150.0 & 25.0 & $-1000.3496412-1000.3497422$ & 52.6 & 8.8 & 52.7 & 8.8 \\
\hline 160.0 & 26.7 & $-1000.3379241-1000.3379903$ & 60.0 & 10.0 & 60.0 & 10.0 \\
\hline 170.0 & 28.3 & $-1000.3253864-1000.3254172$ & 67.8 & 11.3 & 67.9 & 11.3 \\
\hline 180.0 & 30.0 & $-1000.3120165-1000.3120185$ & 76.2 & 12.7 & 76.3 & 12.7 \\
\hline
\end{tabular}


Table S19. Distortion energy per benzene and twist angle per benzene for heptacene (B3LYP/6-31G(d)).

\begin{tabular}{|c|c|c|c|c|c|c|}
\hline \multirow{2}{*}{$\begin{array}{c}\text { Angle } \\
\text { (Degrees) }\end{array}$} & \multirow{2}{*}{$\begin{array}{c}\text { Angle/Benzene } \\
\text { (Degrees) }\end{array}$} & $(\mathrm{au})$ & \multicolumn{4}{|c|}{$(\mathrm{kcal} / \mathrm{mol})$} \\
\hline & & $\mathrm{S}_{0 \text { (closed-shell) }}$ & $\Delta \mathrm{S}_{0}$ (closed-shell) & $\begin{array}{c}\Delta \mathrm{S}_{0 \text { (closed-shell) }} \\
\text { /Benzene }\end{array}$ & $\Delta \mathrm{S}_{0 \text { (open-shell) }}$ & $\begin{array}{c}\Delta \mathrm{S}_{0 \text { (open-shell) }} \\
\text { /Benzene }\end{array}$ \\
\hline 0.0 & 0.0 & $-1154.0667232-1154.0694260$ & 0.0 & 0.0 & 0.0 & 0.0 \\
\hline 10.0 & 1.4 & $-1154.0664087-1154.0691128$ & 0.2 & 0.0 & 0.2 & 0.0 \\
\hline 20.0 & 2.9 & $-1154.0654590-1154.0681674$ & 0.8 & 0.1 & 0.8 & 0.1 \\
\hline 30.0 & 4.3 & $-1154.0638762-1154.0665929$ & 1.8 & 0.3 & 1.8 & 0.3 \\
\hline 40.0 & 5.7 & $-1154.0616970-1154.0644267$ & 3.2 & 0.5 & 3.1 & 0.4 \\
\hline 50.0 & 7.1 & $-1154.0589245-1154.0616709$ & 4.9 & 0.7 & 4.9 & 0.7 \\
\hline 60.0 & 8.6 & $-1154.0555103-1154.0582736$ & 7.0 & 1.0 & 7.0 & 1.0 \\
\hline 70.0 & 10.0 & $-1154.0514472-1154.0542268$ & 9.6 & 1.4 & 9.5 & 1.4 \\
\hline 80.0 & 11.4 & $-1154.0467738-1154.0495689$ & 12.5 & 1.8 & 12.5 & 1.8 \\
\hline 90.0 & 12.9 & $-1154.0414798-1154.0442912$ & 15.8 & 2.3 & 15.8 & 2.3 \\
\hline 100.0 & 14.3 & $-1154.0355308-1154.0383567$ & 19.6 & 2.8 & 19.5 & 2.8 \\
\hline 110.0 & 15.7 & $-1154.0289459-1154.0317844$ & 23.7 & 3.4 & 23.6 & 3.4 \\
\hline 120.0 & 17.1 & $-1154.0217420-1154.0245833$ & 28.2 & 4.0 & 28.1 & 4.0 \\
\hline 130.0 & 18.6 & $-1154.0138849-1154.0167175$ & 33.2 & 4.7 & 33.1 & 4.7 \\
\hline 140.0 & 20.0 & $-1154.0053670-1154.0081798$ & 38.5 & 5.5 & 38.4 & 5.5 \\
\hline 150.0 & 21.4 & $-1153.9962076-1153.9989898$ & 44.2 & 6.3 & 44.2 & 6.3 \\
\hline 160.0 & 22.9 & $-1153.9863898-1153.9891254$ & 50.4 & 7.2 & 50.4 & 7.2 \\
\hline 170.0 & 24.3 & $-1153.9758799-1153.9785480$ & 57.0 & 8.1 & 57.0 & 8.1 \\
\hline 180.0 & 25.7 & $-1153.9646925-1153.9672733$ & 64.0 & 9.1 & 64.1 & 9.2 \\
\hline
\end{tabular}

Figure S1. Distortion energy, $\Delta \mathrm{E}$, per benzene ring plotted as a function of twist angle per benzene ring for polyacenes $n=2-7(B 3 L Y P / 6-31 G(d))$. The plot includes values for twist angles of less than $40^{\circ}$ per benzene ring.

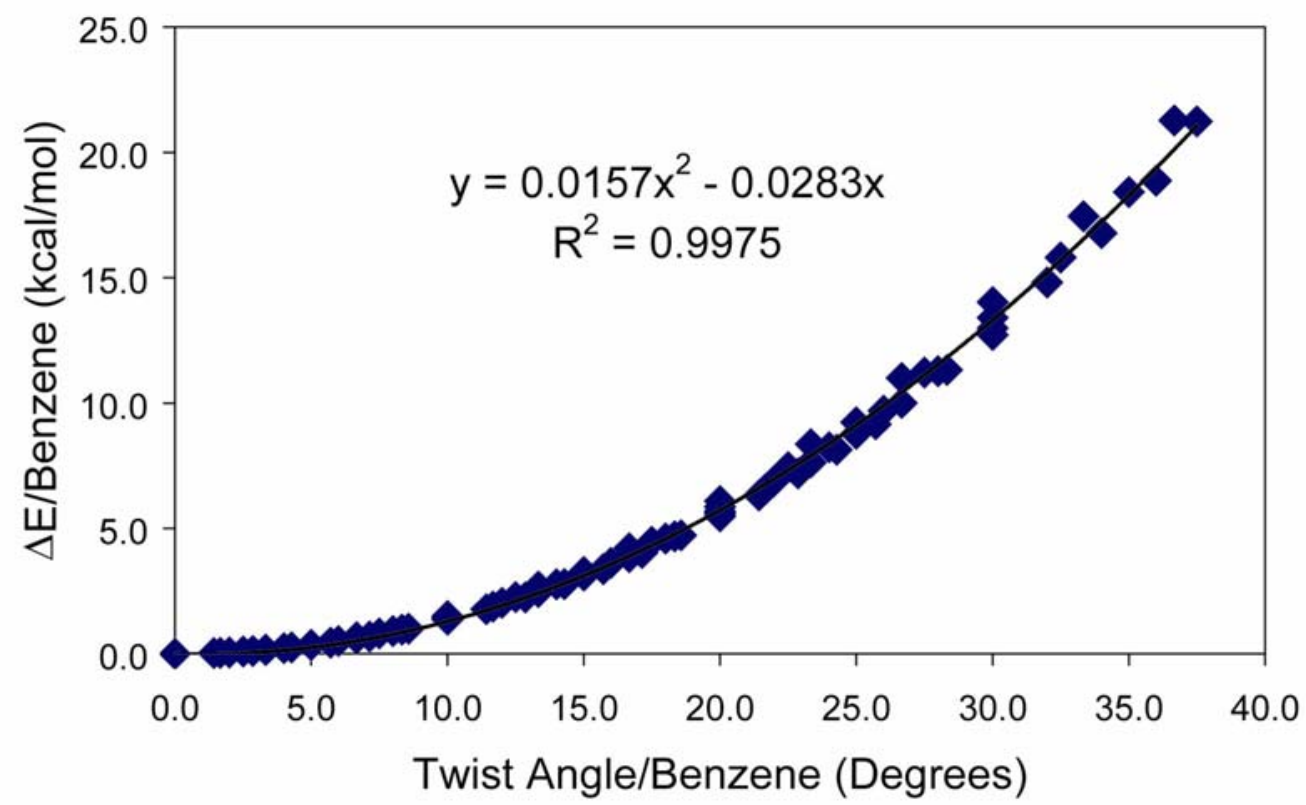

- Polyacenes $(n=2-7)-$ Poly. (Polyacenes $(n=2-7))$ 